\title{
The geometry of the double gyroid wire network: quantum and classical
}

\author{
Ralph M. Kaufmann, Sergei Khlebnikov, and Birgit Wehefritz-Kaufmann
}

\begin{abstract}
Quantum wire networks have recently become of great interest. Here we deal with a novel nano material structure of a double gyroid wire network. We use methods of commutative and noncommutative geometry to describe this wire network. Its noncommutative geometry is closely related to noncommutative 3 -tori as we discuss in detail.
\end{abstract}

Mathematics Subject Classification (2010). 46L60, 81R60, 58B34, 53A10, 57M15, 52C07, 05C38, 81Q10.

Keywords. Double gyroid, noncommutative geometry, K-theory, traces, Harper operator, graph Hamiltonian, noncommutative Brillouin zone, lattices, lattice differential operator, quantum graph, fundamental group, magnetization, projective representations, minimal loops, honeycomb lattice, hexagonal lattice.

\section{Introduction}

Interfaces that can be modeled by surfaces of constant mean curvature (CMC) are ubiquitous in nature and can now be synthesized in laboratory. Recently, Urade et al. [23] have reported fabrication of a nano-porous silica film whose structure is related to a specific CMC surface: the gyroid. The structure is three-dimensionally periodic and has three components: ${ }^{1}$ a thick surface and two channels, as detailed below. The interface between the wall and the channels approximates a double gyroid.

Urade et al. [23] have also demonstrated a nanofabrication technique in which the channels are filled with a metal, while the silica wall can be either left in place or removed. These novel materials open a wide field of applications due to their topological and geometric features. The channels are a few nanometers wide and, when filled with a conducting or semiconducting material, are expected to acquire certain characteristics of one-dimensional quantum wires (such as a blueshift of the spectrum and an enhanced density of states), while remaining three-dimensional in other respects [13]. Geometrically, the one-dimensional structure appears since each of the channels can be retracted to a skeletal graph [8], the gyroid graph.

\footnotetext{
${ }^{1}$ And so is sometimes referred to as tri-continuous.
} 
We will concentrate on the resulting geometry and topology of these networks in the possible presence of a constant magnetic field. Topological quantities are of particular physical interest as they remain stable under continuous perturbations. In practice, those should be small as not to break the structure.

We use two approaches: the first is purely classical and the second is the quantum/noncommutative approach due to Alain Connes [5]. The classical results we provide are a study of the fundamental group of the channel. Here we determine the fundamental group of each channel system. This group is the commutator subgroup of the free group in three generators. A surprising result coming directly from the gyroid geometry is that there is a new length function on the free group which is different from the ordinary word length. With the help of this length function we determine the shortest loops at any given point. There are 15 such topologically distinct shortest loops (30 if one includes orientation). They split into two groups which are distinguished by their cyclic symmetry which is either of order two or three. In both groups there are three generators using this additional symmetry. These loops are of particular interest since numerical simulations [13] show the possibility of an enhanced density of states in a double-gyroid quantum wire, due to states that are nearly localized near such loops. We also calculate the flux of a constant magnetic field through these loops. The tool is an effective unit vector. The result of the calculation is that these effective unit vectors have a particularly simple form (see Table 1). This fact should also be relevant for the study of the spin-orbit coupling of the loop-localized states.

Our study of the noncommutative geometry is motivated by one of the big early successes of the noncommutative geometry of Alain Connes. This was the description by Bellissard et al. of the quantum Hall effect [3]. It allowed to explain the integer effect in terms of the noncommutative geometric properties. The underlying geometry in that situation is the quantum 2-torus. Recently there have been further analyses on the fractional effect using hyperbolic geometry as a model [15]. The conceptual approach as outlined in [2] is to replace the Brillouin zone by a noncommutative Brillouin zone which is given by a $\mathrm{C}^{*}$-algebra that contains the translational symmetry operators and the Hamiltonian. In this geometry relevant quantities can often be expressed in terms of the K-theory of this algebra. This Abelian group captures information related to the topology or better the homotopy type of the algebra or geometric setup. These are again quantities which are stable under continuous deformations. A prime example is the Hall conductance.

A general fact, which is pertinent to our discussion, is that the $K$-groups also serve to label the gaps in the spectrum. Roughly this goes as follows. Given a gap in the spectrum there is a projector projecting to the energy levels below the gap. This projector in turn gives rise to a K-theory element. If one knows the ordered $\mathrm{K}$-theory, then one can also sometimes deduce if only finitely or infinitely many gaps are possible.

In our situation, we determine the said $\mathrm{C}^{*}$-algebra for one channel in the presence of a magnetic field and describe the K-theories. The Hamiltonian we use is the 
generalization [20], [2] of the Harper Hamiltonian [12], [2] adapted to our situation. ${ }^{2}$ We call the resulting algebra the Bellissard-Harper algebra and denote it by $\mathscr{B}$. This geometry is closely related to the noncommutative 3-torus $\mathbb{T}_{\Theta}^{3}$. Here $\Theta$ is a skewsymmetric $(3 \times 3)$-matrix determined by the magnetic field. In fact we show that the algebra is isomorphic to a subalgebra of the $(4 \times 4)$-matrix algebra with coefficients in the noncommutative 3-torus. By varying the magnetic field, we obtain a threeparameter family of algebras. We prove that at all but finitely many points this algebra is the full matrix algebra and hence Morita equivalent to $\mathbb{T}_{\Theta}^{3}$. Since $\mathbb{T}_{\Theta}^{3}$ is simple at irrational $\Theta$, one would expect this to be true on a dense set (see $\S 3$ ). We not only prove that this expectation holds at the irrational points, but we are able to extend this result to almost all rational points. At certain special values of the field which we enumerate, however, the algebra is genuinely smaller, leading to a possibly different K-theory. At these points the material may also exhibit special properties.

The ordered K-theory of the 3-torus is completely known [18], [17], [6] and actually completely classifies the isomorphism classes of such tori [19], [7]. Our algebra always injects into a $\mathbb{T}_{\Theta}^{3}$. From this and the knowledge of the ordered $K$ group of the noncommutative torus, we obtain the result that there are only finitely many gaps possible at rational values of $\Theta$.

Our approach to both the classical and the quantum geometry uses graph-theoretical methods. The relevant graphs are the gyroid graph which is a 3-regular graph and its quotient under the translation symmetry group. In order to give the matrix algebras explicitly we introduce the notions of a graph representation. Using rooted spanning trees, we are able to represent the algebra $\mathcal{B}$ in terms of matrices, where they are amenable to direct computations.

As the geometry of the gyroid and its channels is quite difficult, we also treat the honeycomb lattice as a two-dimensional analogue. Indeed, the gyroid lattice graph (the graph onto which each channel retracts) is in many ways the three-dimensional analogue of the honeycomb lattice, which is why when developing the more general parts of the theory we will in parallel consider these two cases as our main examples. Both graphs are 3-regular, and both of the graphs are not mathematical lattices but only physical lattices. ${ }^{3}$ This means that they give rise to two groups, one which is the space or symmetry group and the other is the group of the lattice, of which they can be considered a subset. In the honeycomb case these are both $\mathbb{Z}^{2}$ but embedded into $\mathbb{R}^{2}$ as the triangular lattice and its dual. For the gyroid the groups are both $\mathbb{Z}^{3}$, but they are embedded as a body centered cubic (bcc) and a face centered cubic (fcc) lattice in $\mathbb{R}^{3}$, which are again dual to each other. Furthermore the fundamental group of the honeycomb lattice is the commutator subgroup of the free group on two elements, while for the gyroid $\pi_{1}$ is the commutator subgroup of the free group in three generators. And in both cases we find a new length function on the free group induced by the geometry of the lattice.

\footnotetext{
${ }^{2}$ The generalized Harper operator is also the operator underlying the noncommutative geometry of the quantum Hall effect [3], [15].

${ }^{3}$ See $\$ 3.2$ for the disambiguation of the use of the word "lattice".
} 
The paper is organized as follows:

In the first section, we start with a review of the classical geometry of the gyroid and then prove the results on the fundamental group and the smallest loops. In the second section, we formally introduce graphs and lattices and the relevant groups associated to them. We then define their representation in Hilbert spaces and the associated Harper operators. Finally, we show how to obtain a matrix representation of $\mathscr{B}$ using rooted spanning trees. In the third section, we apply the general theory of the second section to the noncommutative geometry of a lattice using a Harper operator, detailing the honeycomb and the gyroid case. Here we also briefly review projective representations and explain how they arise in the presence of a magnetic field. In this context, we can already show that there can only be finitely many gaps for the gyroid at rational $\Theta$. We also outline the general approach to calculating $\mathscr{B}$ and its K-theory and the expected results. In particular, we compute the K-theory in the commutative case in terms of a cover of a torus.

In the last section we apply the outlined strategies to calculate the algebras $\mathscr{B}$ and their K-theory for Bravais lattices, the honeycomb lattice and the gyroid lattice graph.

\section{The classical geometry of the double gyroid}

1.1. The double gyroid and its channels. The gyroid is an embedded CMC surface in $\mathbb{R}^{3}$ [9]. It was discovered by Alan Schoen [21]. In nature it was observed as an interface for di-block co-polymers [10], [22]. The interface actually consists of two disconnected surfaces. Each of them is a gyroid surface. The double gyroid (DG) is a particular configuration of two mutually non-intersecting embedded gyroids.

A single gyroid has symmetry group $I 4_{1} 32$ while the double gyroid has the symmetry group $I a \overline{3} d$ where the extra symmetry comes from interchanging the two gyroids. ${ }^{4}$

Since CMC surfaces are mathematically hard to handle level surfaces have been suggested as a possible approximation in [14]. The level surface approximation for the double gyroid again consists of two level surfaces. We will call the two surface interfaces $S_{1}$ and $S_{2}$. For the discussion at hand it is not relevant if the two surfaces are actually the $\mathrm{CMC}$ surfaces or their level surface approximations. One example of a DG approximation is given by the family of level surfaces [14]

$$
L_{t}: \sin x \cos y+\sin y \cos z+\sin z \cos x=t .
$$

A model for the double gyroid is then given by $L_{w}$ and $L_{-w}$ for $0 \leq w<\sqrt{2}$.

The complement $\mathbb{R}^{3} \backslash G$ of a single gyroid $G$ has two components. These components will be called the gyroid wire systems or channels.

\footnotetext{
${ }^{4}$ Here $I 4_{1} 32$ and $I a \overline{3} d$ are given in the international or Hermann-Mauguin notation for symmetry groups; see e.g. [11].
} 
There are two distinct channels, one left and one right handed. Each of these channels contracts onto a graph, called skeletal graph in [21], [8]. We will call these graphs $\Gamma_{+}$and $\Gamma_{-}$. Each graph is periodic and trivalent. We fix $\Gamma_{+}$to be the graph which has the node $v_{0}=\left(\frac{5}{8}, \frac{5}{8}, \frac{5}{8}\right)$. We will give more details on the graph $\Gamma_{+}$below.

The channel containing $\Gamma_{+}$is shown in Figure 1. A (crystal) unit cell of the channel together with the embedded graph $\Gamma_{+}$is shown in Figure 2 and just the graph is contained in Figure 3.

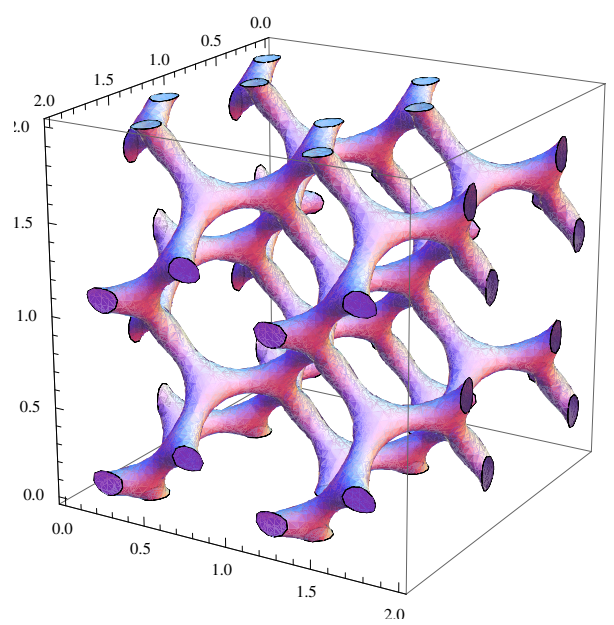

Figure 1. The channel $C_{+}$.

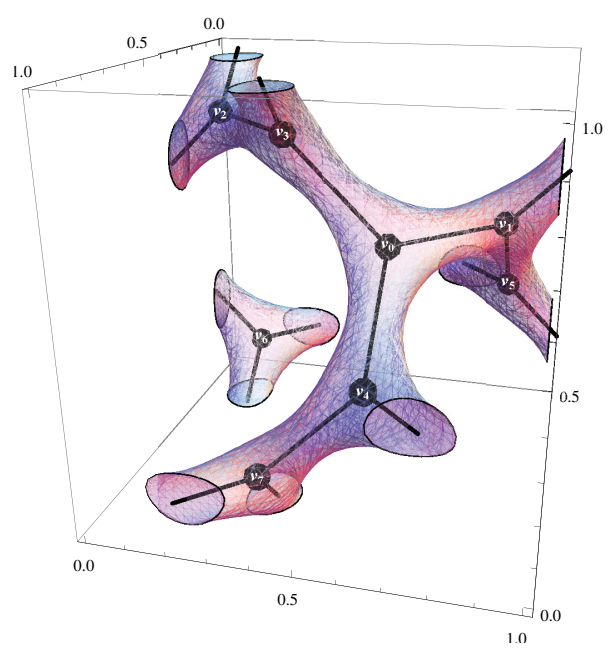

Figure 2. The graph $\Gamma_{+}$embedded into its channel $C_{+}$. 


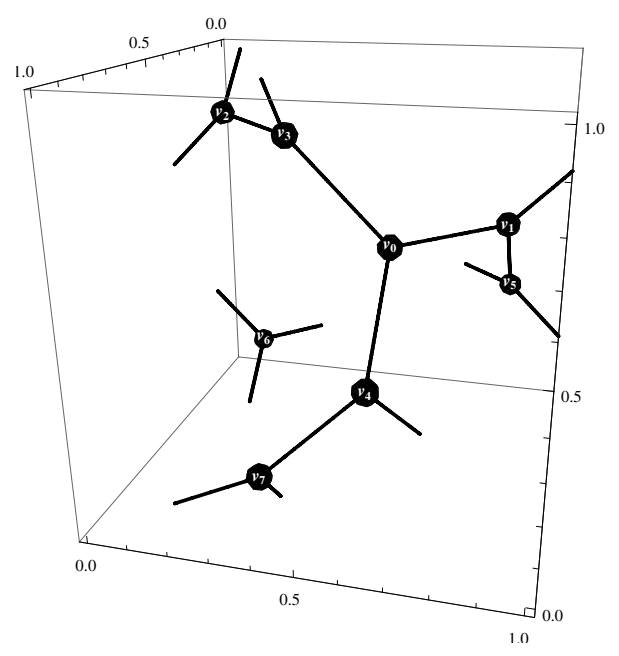

Figure 3. The graph $\Gamma_{+}$.

In constructing the surface, one can actually start with one of these graphs and evolve the surface from it [8]. The symmetry of one skeletal graph is that of a single gyroid $I 4_{1} 32$.

To obtain the double gyroid one can evolve from both the graphs. The common symmetry group of both of these graphs is $I a \overline{3} d$. This symmetry group can actually be defined already on the nodes $V_{ \pm}$of the two graphs $\Gamma_{ \pm}$. The subgroup $I 4_{1} 32$ is then determined to be the subgroup that fixes both sets of nodes (setwise).

In the case of the double gyroid $S=S_{1} \amalg S_{2}$, the complement $C=\mathbb{R}^{3} \backslash S$ of the disjoint union of the two gyroid surfaces has three connected components. These are two channel systems $C_{+}$and $C_{-}$, each of which can be retracted to its skeletal graph $\Gamma_{ \pm}$. In fact, the skeletal graph is a deformation retract [8]. There is a third connected component $F$ and we will write $\bar{F}=F \cup S$. This is a 3-manifold with two boundary components, more precisely, $\partial \bar{F}=S=S_{1} \amalg S_{2}$. F can be thought of as a "thickened" (fat) surface. The thickness is fixed by the parameter $w$. Note that $F$ can be retracted to each of the two boundary surfaces $S_{i}$. In fact there is a deformation retract of $F$ onto the gyroid.

This means that all homotopical information about the complements of the double gyroid is encoded in the gyroid/level surface and the two skeletal graphs.

The translational symmetry group for both the gyroid and the double gyroid is the Bravais lattice bcc. Note that we will usually use "lattice" in the physical terminology, e.g. speak about the honeycomb lattice. The term "Bravais lattice" will be used to denote a maximal rank mathematical lattice, i.e., a free rank $n$ Abelian subgroup of $\mathbb{R}^{n}$. In order to preempt any confusion, we give precise definitions for our terminology in $\$ 3.2$. 


\subsection{The skeletal graph}

1.2.1. The vertices and edges. We will now describe the graph $\Gamma_{+}$embedded into $\mathbb{R}^{3}$.

Set

$$
\begin{array}{ll}
v_{0}=\left(\frac{5}{8}, \frac{5}{8}, \frac{5}{8}\right), & v_{4}=\left(\frac{7}{8}, \frac{5}{8}, \frac{3}{8}\right), \\
v_{1}=\left(\frac{3}{8}, \frac{7}{8}, \frac{5}{8}\right), & v_{5}=\left(\frac{1}{8}, \frac{7}{8}, \frac{3}{8}\right), \\
v_{2}=\left(\frac{3}{8}, \frac{1}{8}, \frac{7}{8}\right), & v_{6}=\left(\frac{1}{8}, \frac{1}{8}, \frac{1}{8}\right), \\
v_{3}=\left(\frac{5}{8}, \frac{3}{8}, \frac{7}{8}\right), & v_{7}=\left(\frac{7}{8}, \frac{3}{8}, \frac{1}{8}\right),
\end{array}
$$

and let $\bar{V}_{+}=\left\{v_{0}, \ldots, v_{7}\right\}$.

Furthermore $\mathbb{Z}^{3}$ acts on $\mathbb{R}^{3}$ by translations and we let $V_{+}=\mathbb{Z}^{3}\left(\bar{V}_{+}\right)$be the image of the set $\bar{V}_{+}$under this action. We will sometimes call this set of points the gyroid lattice.

Given two points $v, w \in \mathbb{R}^{3}$ let $\overline{v w}=\{(1-t) v+t w \mid t \in[0,1]\}$ be the line segment joining them. Also given a point $v$ we let $T_{x}(v)=v+(1,0,0)$, $T_{y}(v)=v+(0,1,0), T_{z}(v)=v+(0,0,1)$ be the translated points.

Consider the following set of line segments

$$
\begin{gathered}
\bar{E}=\left\{\overline{v_{0} v_{1}}, \overline{v_{0} v_{3}}, \overline{v_{0} v_{4}}, \overline{v_{2} v_{3}}, \overline{v_{4} v_{7}}, \overline{v_{1} v_{5}},\right. \\
\overline{v_{4} T_{x}\left(v_{5}\right)}, \overline{v_{7} T_{x}\left(v_{6}\right)}, \overline{v_{1} T_{y}\left(v_{2}\right)}, \\
\left.\overline{v_{5} T_{y}\left(v_{6}\right)}, \overline{v_{2} T_{z}\left(v_{6}\right)}, \overline{v_{3} T_{z}\left(v_{7}\right)}\right\} .
\end{gathered}
$$

Let $E_{+}=\mathbb{Z}^{3}(\bar{E})$, where again $\mathbb{Z}^{3} \subset \mathbb{R}^{3}$ acts as a subgroup of the translation group.

Definition 1.1. The graph $\Gamma_{+}$is the graph whose vertices are $V_{+}$, whose edges are $E_{+}$with the obvious incidence relations.

We recall:

Proposition 1.2 ([8]). $C_{+}$can be deformation retracted onto the graph $\Gamma_{+}$and $\Gamma_{+}$ is the component of the critical graph contained in $C_{+}$.

Corollary 1.3. The homotopy type of the complement $T=\mathbb{R}^{3} \backslash G$ is the same as that of two copies of $\Gamma_{+}$. In particular each channel has the same homotopy type as $\Gamma_{+}$.

This implies that all topological invariants $C_{ \pm}$which are homotopy invariant are isomorphic to those of $\Gamma_{+}$. In particular, this means all homology, homotopy and $\mathrm{K}$-groups of the topological space $C_{+}$or $C_{-}$are determined on $\Gamma_{+}$. 
1.3. The quotient graphs. Quotienting out by either the standard translation group or the bcc lattice, we obtain the following two quotient graphs.

1.3.1. Crystallographic quotient graph. Let $\bar{\Gamma}_{+}^{\text {crystal }}$ be the graph $\Gamma_{+} / \mathbb{Z}^{3}$ thought of as an abstract graph. This graph has a natural map embedding into the 3-torus $\mathbb{R}^{3} / \mathbb{Z}^{3}$.

Proposition 1.4. $\bar{\Gamma}_{+}^{\text {crystal }}$ is a cube embedded into the 3-torus. More precisely the vertices of $\bar{\Gamma}_{+}^{\text {crystal }}$ are $\bar{V}$ and the edges are the images of $\bar{E}$ in $\mathbb{R}^{3} / \mathbb{Z}^{3}$.

The abstract graph is given in Figure 4 which also contains the images of the vectors $e_{i}$ which name and orient all edges.

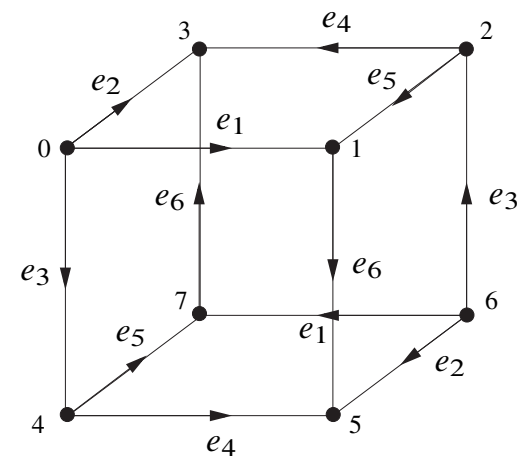

Figure 4. The graph $\bar{\Gamma}_{+}^{\text {crystal }}$ for the gyroid.

Proof. Since all the vertices in the fundamental domain do not lie on the boundary, they give exactly the representatives of $V$ in $\mathbb{R}^{3} / \mathbb{Z}^{3}$. The classes of the edges $E_{\Gamma_{+}}$ are given exactly by the images of the set $\bar{E}$ in $\mathbb{R}^{3} / \mathbb{Z}^{3}$.

1.3.2. The (maximal) quotient graph. By modding out by $\mathbb{Z}^{3}$, we have not yet used the full translational symmetry of $\Gamma_{+}$which is the bcc lattice. A set of generators for the bcc lattice is

$$
f_{1}:=(1,0,0), \quad f_{2}=(0,1,0), \quad f_{3}:=\frac{1}{2}(1,1,1)
$$

another set of generators which is more symmetric and we will use later on is:

$$
g_{1}=\frac{1}{2}(1,-1,1), \quad g_{2}=\frac{1}{2}(-1,1,1), \quad g_{3}=\frac{1}{2}(1,1,-1) .
$$

We let $L=L\left(\Gamma_{+}\right)$be the free Abelian subgroup of $\mathbb{R}^{3}$ generated by these vectors. We define $\bar{\Gamma}_{+}$to be the abstract quotient graph $\Gamma_{+} / L$. 
Proposition 1.5. $\bar{\Gamma}_{+}$is the graph with four vertices and six edges, where all pairs of distinct vertices are connected by exactly one edge.

This graph is sometimes also called the complete square. Its incidence matrix has entries one everywhere except on the diagonal, where the entries are zero. This graph is shown in Figure 5.
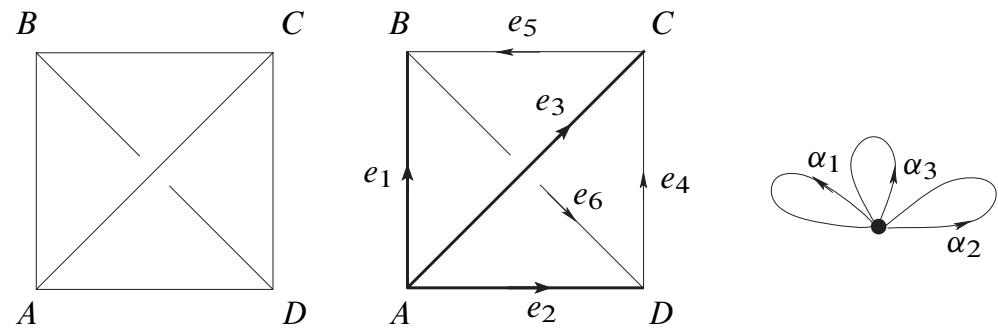

Figure 5. The complete square, the rooted spanning tree $\tau$ (root $A$ and edges $e_{1}, e_{2}, e_{3}$ ) and the vectors corresponding to the oriented edges, the collapsed tree $\bar{\Gamma}_{+} / \tau$.

Proof. We see that there is an embedding of $\mathbb{Z}^{3} \subset L$, where $L$ is the bcc lattice, so that we only have to divide $\bar{\Gamma}_{+}^{\text {crystal }}$ by the additional symmetry generated by $d:=$ $\frac{1}{2}(1,1,1)$. Now $\bmod \mathbb{Z}^{3}, T_{d}^{2} \cong \mathrm{id}$ and $T_{d}$ (the translation by $d$ ) simply interchanges the vertices of $\bar{\Gamma}_{+}^{\text {crystal }}$ as follows: $v_{0} \leftrightarrow v_{6}, v_{1} \leftrightarrow v_{7}, v_{2} \leftrightarrow v_{4}$ and $v_{3} \leftrightarrow v_{5}$. Hence we are left with four vertices, and we can choose the representatives $v_{0}, \ldots, v_{3}$. Checking the list of edges (1) one sees that indeed the twelve edges form pairs and one can choose the representatives $\overline{v_{i} v_{j}} ; i \neq j, i, j=0, \ldots, 3$.

Notice that this corresponds to a $\mathbb{Z} / 2 \mathbb{Z}$ symmetry of the graph $\bar{\Gamma}_{+}^{\text {crystal }}$. It is given by mapping each vertex to its diagonally opposite vertex and maps the edges accordingly.

1.4. The underlying group and lattice. There is another Bravais lattice hidden in the geometry of the gyroid. This is the fcc lattice. The nearest neighbor positions differ by vectors generating an fcc lattice.

This means in particular that after shifting by $v_{0}$ the positions of the vertices of $\Gamma_{+}$all lie on an fcc lattice.

In order to fix notation set

$$
\begin{array}{lll}
e_{1}=v_{1}-v_{0}, & e_{2}=v_{3}-v_{0}, & e_{3}=v_{4}-v_{0}, \\
e_{4}=v_{3}-v_{2}, & e_{5}=v_{7}-v_{4}, & e_{6}=v_{5}-v_{1} .
\end{array}
$$


Definition 1.6. Let $T\left(\Gamma_{+}\right)$be the group of $\mathbb{R}^{3}$ that is generated by

$$
\begin{array}{ll}
e_{1}=\frac{1}{4}\left(\begin{array}{c}
-1 \\
1 \\
0
\end{array}\right), \quad e_{2}=\frac{1}{4}\left(\begin{array}{c}
0 \\
-1 \\
1
\end{array}\right), \quad e_{3}=\frac{1}{4}\left(\begin{array}{c}
1 \\
0 \\
-1
\end{array}\right) \\
e_{4}=\frac{1}{4}\left(\begin{array}{l}
1 \\
1 \\
0
\end{array}\right), \quad e_{5}=\frac{1}{4}\left(\begin{array}{c}
0 \\
-1 \\
-1
\end{array}\right), \quad e_{6}=\frac{1}{4}\left(\begin{array}{c}
-1 \\
0 \\
-1
\end{array}\right) .
\end{array}
$$

Proposition 1.7. The group $T\left(\Gamma_{+}\right)$is isomorphic to $\mathbb{Z}^{3}$. The Bravais lattice it generates is a face centered cubic $(f c c)$.

Proof. There are relations among the $e_{i}$ given by

$$
e_{1}=-e_{5}+e_{6}, \quad e_{2}=-e_{4}-e_{6}, \quad e_{3}=e_{4}+e_{5}
$$

so that we see that $e_{3}, e_{4}, e_{5}$ are generators. These vectors are linearly independent over $\mathbb{Z}$ since they are independent over $\mathbb{R}$. Hence they provide a free basis and an isomorphism to $\mathbb{Z}^{3}$. The vectors $e_{4},-e_{5},-e_{6}$ are the standard primitive vectors for the face centered cubic.

Of course there are many other choices of basis here, e.g. $\left\{e_{2}, e_{4}, e_{6}\right\}$.

Proposition 1.8. The vertices of $\Gamma_{+}$translated by $-v_{6}$ lie on the three-dimensional face centered cubic lattice generated by $e_{4}, e_{5}, e_{6}$.

Proof. $\Gamma_{+}$is path connected and we take $v_{6}$ as the base point. Each line segments in $E$ corresponds to an edge. Choosing an orientation for this edge defines a vector. Now the statement follows from the fact that the vectors corresponding to the line segments in $E$ and hence those of $E_{\Gamma_{+}}$are exactly the vectors $\pm e_{1}, \ldots, \pm e_{6}$.

1.5. Fundamental groups, loops and effective normal vectors. There is certain geometric information which can even already be read off from the simple graph $\bar{\Gamma}_{+}$. Such as its fundamental group or the minimal loops starting at a given vertex. A loop on $\Gamma_{+}$is minimal if it passes through a minimal number of edges. A more general treatment will be given in $\S 3$.

Proposition 1.9. Let $\mathbb{F}_{3}$ be the free group in three variables. The (realization) of the graphs $\Gamma_{+}$and $\bar{\Gamma}_{+}$have following fundamental groups:

(1) $\pi_{1}\left(\Gamma_{+}\right)=\left[\mathbb{F}_{3}, \mathbb{F}_{3}\right]$,

(2) $\pi_{1}\left(\bar{\Gamma}_{+}\right)=\mathbb{F}_{3}$.

In particular $\Gamma_{+}$is the maximal Abelian cover of $\bar{\Gamma}_{+}$.

Since $\Gamma_{+}$is homotopic to one channel, these results hold for each channel of the gyroid. 
Proof. We start with $\bar{\Gamma}_{+}$. This graph is homotopic to the wedge product of three $S^{1}$,s, whence the second claim follows.

In view of Lemma 1.10 below (more generally by Proposition 3.5), we see that $\pi_{1}\left(\Gamma_{+}\right)$is the subgroup that is the kernel of the map $\mathbb{F}_{3}$ to its Abelianization. Indeed the sum of powers of each of the generators in a word in the group has to be zero, which precisely means that such a word is in the kernel of the Abelianization map or in other words, the commutator group.

Lemma 1.10. A loop on the graph $\bar{\Gamma}_{+}$lifts to a loop on $\Gamma_{+}$if and only if each edge is traversed the same number of times in each direction.

Proof. The "if" direction is clear since this means that the translations have to add up to zero. The equivalence follows from the general Proposition 3.5 by noticing that indeed the lifts $l_{1}=e_{2} e_{6}^{-1} e_{1}^{-1}, l_{2}=e_{1} e_{5}^{-1} e_{3}^{-1}, l_{3}=e_{3} e_{4} e_{2}^{-1}$ give rise to the vectors $\vec{l}_{i}=f_{i}$ of (3) and are linearly independent.

Proposition 1.11. There are closed loops in the graph $\Gamma_{+}$. Each minimal loop goes through 10 sites and at each point there are 30 oriented minimal loops or 15 such undirected loops.

Proof. A path in which one goes back and forth through an edge is homotopic to the path in which this step is omitted. By direct calculation one can see that such a path is given by traversing any five of the six edges. At the first step one has three choices of edges, at the second step there are two and then again two possibilities. Here one either returns to the original vertex or not. In the first case there are 2 completions, and in the second case three completions to a minimal loop. Thus we have $2 \cdot 3 \cdot 5=30$ possibilities. A detailed version of this enumeration is provided at the end of this section in §1.5.1.

1.5.1. Explicit calculation of the loops. Here we give the details of the calculation of the loops. Using Proposition 3.5, we have to look for paths that traverse each edge the same number of times. We first look at the cases where each edge is traversed only once in each direction. We call such a path a good path. We also have to keep the number of these edges minimal. This already puts a simple constraint on the path. We may not go back and forth through the same edge. Starting at the given vertex we have to pass through two distinct edges. After this we have a choice, we can either go back to the starting vertex (case I) or we can go to the only vertex which we have not reached yet (case II). In case I the next oriented edge is fixed, but then we have two choices Ia and Ib. After this we have already used five edges so that the minimal possible number of oriented edges and hence the length of the loop is 10. Indeed, we can complete the edge path uniquely to a good path without traversing the 6th edge.

In case II the fifth oriented edge for a good path is fixed, going opposite the first oriented edge. There is a choice for the sixth oriented edge in the path. Either 
returning to $v_{o}$ (IIb) or not (IIa). The case IIb has a unique choice for a fifths oriented edge for a good path and this has a unique completion to a good path involving five edges, again giving a loop of length 10. In case IIa, we again have two choices for the fifth oriented edge (IIa1) and (IIa2). Both these choices have a unique completions to good paths again of length 10 .
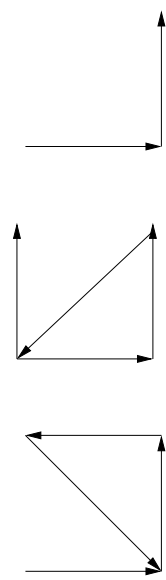

IIa

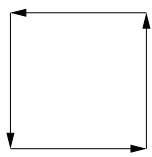

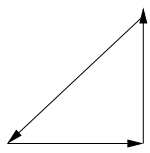

I
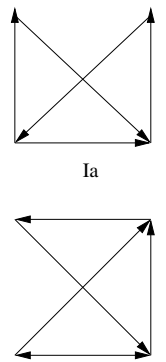

IIal

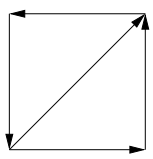

IIb
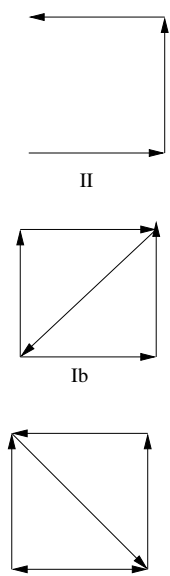

IIa2

Figure 6. The combinatorial cases for the minimal loops of the gyroid.

It remains to treat the cases where an edge is traversed more that once in each direction. Since we are not allowed to go back and forth on one edge the choices for the first three oriented edges are as above. Now in case I we could go along the first oriented edge again, but this would lead us to traverse at least six edges and hence would not be minimal. At the next step of case I the choices are precisely Ia or Ib and traversing an edge twice in the same direction would not be minimal. For case II, the first stage where one could use an oriented edge twice for IIa is the fifth edge. But then one would need at least six edges counting multiplicities. After fixing the fifth oriented edge one can only increase the number of traversed oriented edges by not choosing a good path. Finally in case IIb again the first edge with a choice to traverse an edge twice in one orientation is at the fifth oriented edge, but as before this would lead to a path of length greater than 10 .

So all in all we have $2 \cdot 3=6$ choices for the first two edges and then once these are fixed 5 choices for a good path. This means in all there are 30 such oriented paths. 
1.5.2. Explicit loops. On the graph $\bar{\Gamma}_{+}$there is a symmetry group of order 6 preserving the base point and the spanning tree $\tau$ which permutes the vertices $B, C$ and $D$. This is precisely the group that gives us the six first choices.

In order to write down a shorter list, we will make the following observations. Since the inverse of a minimal path is a minimal path, we can cut down the number to 15 . Now since each of the paths traverses five edges, it misses one. There are two cases: (1) the edge that is missed is incident to $v_{0}$, i.e., $e_{1}, e_{2}$ or $e_{3}$, and in case (2) it is not, i.e., $e_{3}, e_{4}, e_{6}$. Case (1) corresponds to IIa1 and IIa2, which case (2) corresponds to Ia, Ib and IIb. In case (1) the vertex $v_{0}$ is traversed one additional time except at the start and end of the path and in case (2) it is traversed an additional two times. This decomposes the loops into two pieces of length 5 in case (1) or three pieces of length 3, 3, 4; 3, 4, 3; 4, 3, 3 in case (2) for Ia, Ib, IIb respectively. With each loop, its cyclic permutation of these components is also a loop. There are 2 such loops in case (1) and 3 such loops in case (2). This also explains the 15 loops as $15=3 \cdot 2+3 \cdot 3$ and permutes the cases IIa1, IIa2; and Ia, IIb and Ib cyclically.

We give here a list of 6 basic loops which may be useful for further discussion. To obtain all 30 one should cyclically permute the blocks and take full inverses of the loops. We give the loops as an edge path as well as their decomposition into the basis loops $l_{1}, l_{2}, l_{3}$.

\begin{tabular}{|c|c|c|c|}
\hline loop as an edge path in the $e_{i}$ 's & in basis $l_{i}$ & $N_{\text {eff }}$ & edge missed \\
\hline$e_{2}\left(-e_{4}\right) e_{5} e_{6}\left(-e_{2}\right) e_{3} e_{4}\left(-e_{6}\right)\left(-e_{5}\right)\left(-e_{3}\right)$ & $\left(l_{1} l_{2} l_{3}\right)^{-1}\left(l_{1} l_{2} l_{3}\right)$ & $(1,-1,0)$ & $e_{1}$ missing \\
\hline$e_{1}\left(-e_{5}\right) e_{4}\left(-e_{6}\right)\left(-e_{1}\right) e_{3} e_{5} e_{6}\left(-e_{4}\right)\left(-e_{3}\right)$ & $\left(l_{2} l_{3} l_{1}\right)\left(l_{3} l_{1} l_{2}\right)^{-1}$ & $(0,1,-1)$ & lissing \\
\hline$e_{1}\left(-e_{5}\right) e_{4}\left(-e_{6}\right)\left(-e_{1}\right) e_{2}\left(-e_{4}\right) e_{5} e_{6}\left(-e_{2}\right)$ & $\left(l_{2} l_{3} l_{1}\right)\left(l_{1} l_{2} l_{3}\right)^{-1}$ & $(1,0,-1)$ & $e_{3}$ missing \\
\hline$e_{1}\left(-e_{5}\right)\left(-e_{3}\right) e_{2}\left(-e_{6}\right)\left(-e_{1}\right) e_{3} e_{5} e_{6}\left(-e_{2}\right)$ & {$\left[l_{2}, l_{1}\right]$} & $(1,1,0)$ & $e_{4}$ missing \\
\hline$e_{1} e_{6}\left(-e_{2}\right) e_{3} e_{4}\left(-e_{6}\right)\left(-e_{1}\right) e_{2}\left(-e_{4}\right)\left(-e_{3}\right)$ & {$\left[l_{1}^{-1}, l_{3}\right]$} & $(0,-1,-1)$ & $e_{5}$ missing \\
\hline$e_{1}\left(-e_{5}\right) e_{4}\left(-e_{2}\right) e_{3} e_{5}\left(-e_{1}\right) e_{2}\left(-e_{4}\right)\left(-e_{3}\right)$ & {$\left[l_{2}, l_{3}\right]$} & $(-1,0,-1)$ & $e_{6}$ missing \\
\hline
\end{tabular}

Table 1. A generating set the shortest loops and their effective normal vectors.

Remark 1.12. It is interesting to note that the explicit isomorphism $\mathbb{F}_{3} \rightarrow \pi_{1}\left(\bar{\Gamma}_{+}\right)$ given on the generators $\alpha_{i}$ of $\mathbb{F}_{3}$ by $\alpha_{i} \mapsto l_{i}$ induces a new length on $\mathbb{F}_{3}$ as the minimal length in the letters $e_{i}$. It is this length that remembers the structure of the gyroid. The computation above is a good example of this. The three examples of commutators are minimal elements of $\left[\mathbb{F}_{3}, \mathbb{F}_{3}\right]$ which are not the identity in the length in $\alpha_{i}$ or $l_{i}$. They are of length 4 . In the $e_{i}$ their length is 10 . The other examples are of length 6 in the $\alpha_{i}$, so not minimal in this metric, but they are minimal in the $e_{i}$ being of length 10 .

1.5.3. Effective normal vector. One distinguishing feature of the different loops is their spatial orientation. This for instance has an effect on the flux through a surface bounded by such a loop. 
We assume a constant magnetic field. In this case if $S$ is a surface bounding a loop $L=e_{i_{1}}, \ldots, e_{i_{n}}$, let $f_{j}:=\sum_{k=1}^{j} e_{i_{j}}$. Then, by Stokes, we can just integrate over the surface given by the union of the triangles defined by $\left(f_{j}, f_{j+1}\right), j=1, \ldots, n-1$. If $N_{j}=f_{j} \times f_{j+1}=f_{j} \times e_{j+1}$ and $N_{\text {eff }}:=\sum_{j=1}^{n-1} N_{j}$, then we have for the magnetic flux through $S$

$$
\Phi=\iint_{S} B d S=\sum_{j} \frac{1}{2} B \cdot N_{j}=\frac{1}{2} B \cdot N_{\mathrm{eff}}
$$

The values for $N_{\text {eff }}$ are listed for the basic loops. Notice that inversion of a loop inverts the normal vector, while the cyclic permutation of the components leaves the effective normal vector invariant.

1.6. The quantum graph. In order to promote the skeletal graph to a quantum graph, we will fix a Hilbert space and a Hamiltonian. Here we follow the terminology that a quantum graph is graph with an associated Hilbert space and a Hamiltonian on it. In this paper we will use the generalized Harper Hamiltonian [2], [20].

The original Harper Hamiltonian [12] is obtained for a cubic lattice by using the tight-binding approximation and Peierls substitution for the quasi momentum [16].

In the next section we will give the general theoretical setup for this using graphs, groups and representations. For the gyroid the notions such as graphs and groups have been introduced above, so that the reader may substitute these in the general definitions below.

\section{Graph representations and matrix Harper operators}

One idea in studying the noncommutative aspects of a given system is to give a Ktheoretic gap labeling for the Hamiltonians. For this one considers an algebra $\mathscr{B}$ generated by the Hamiltonian and the symmetries. If everything is commutative then this algebra is basically the $\mathrm{C}^{*}$-algebra of functions on the Brillouin zone/torus. We will make these ideas precise using physical and mathematical lattices as defined below.

2.1. Graph language. For us a graph or an abstract graph $\Gamma$ will be a collection of vertices $V(\Gamma)$ and a collection of edges $E(\Gamma)$ which run between vertices up to bijections preserving the incidences. Each edge can have two orientations. An edge together with an orientation is called an oriented edge. Each oriented edge $\vec{e}$ has a starting vertex $s(\vec{e})$ and a target vertex $t(\vec{e})$. A graph $\Gamma$ is called finite if both $V(\Gamma)$ and $E(\Gamma)$ are finite sets.

A graph naturally becomes a topological space if the edges are replaced by intervals. This space is called the realization of the graph. In more technical terms the data above gives a one-dimensional CW-complex and we take the realization of this 
complex. When we talk about topological properties of a graph, like its fundamental group, we always mean the topological properties of its realization.

A graph is connected if its realization is connected. This means that one can travel to all vertices from any given vertex along the edges. A tree is a connected graph whose realization is contractible (i.e., the graph has no loops). A choice of root of a tree is simply a choice of a vertex and a rooted tree is a tree together with a choice of a root. Given a graph $\Gamma$ a subgraph $\tau$ is called a spanning tree if it is a tree and the vertices of $\tau$ are all of the vertices of $\Gamma$. To have a spanning tree $\Gamma$ needs to be connected. In this case there are usually several choices of spanning trees. A rooted spanning tree is a spanning tree together with the additional choice of a root.

Proposition 2.1. Let $\bar{\Gamma}$ be a finite graph and $\tau$ be a rooted spanning tree. Let $v_{0}$ be the root. Then $\pi_{1}(\bar{\Gamma}):=\pi_{1}\left(\bar{\Gamma}, v_{0}\right)=\mathbb{F}_{n}$, where $\mathbb{F}_{n}$ is the free group in $n$ variables and $n=|E(\bar{\Gamma})|-|E(\tau)|$.

Proof. Consider the graph $\bar{\Gamma} / \tau$ obtained by contracting all edges of the subgraph $\tau$. This is the graph which only has one vertex $v_{0}$ and all the edges are loops. The two graphs $\bar{\Gamma}$ and $\bar{\Gamma} / \tau$ are homotopy equivalent and hence have the same fundamental group. The graph $\bar{\Gamma} / \tau$ embeds into the plane with $n$ punctures with each loop going around one puncture. To obtain a compatible embedding of $\Gamma$, one blows up the only vertex of $\bar{\Gamma} / \tau$ into the tree $\tau$ and both graphs are homotopy equivalent to the punctured plane. $\bar{\Gamma}$ is thus homotopy equivalent to the wedge product of $n$ circles $S^{1}$. It is well known that the first homotopy group of this space is the free group in $n$ generators.

An embedded graph is a graph $\Gamma$ together with an embedding of it realization into an $\mathbb{R}^{n}$. Some properties we discuss depend on such an embedding or are derived from it.

Example 2.2. We have so far considered $\bar{\Gamma}_{+}^{\text {crystal }}$ and $\bar{\Gamma}_{+}$as abstract graphs and we have considered $\Gamma_{+}$both as an abstract as well as an embedded graph. The properties of having a certain number of loops at a given point are properties of the abstract graph, while the proof made use of the embedding. The effective normal vectors are properties of the embedding of the skeletal graph $\Gamma_{+}$into $\mathbb{R}^{3}$.

\subsection{Graph Harper operator}

Definition 2.3. A $C^{*}$-representation $\rho_{\Gamma}$ of a graph $\Gamma$ is given by the following data:

- a collection of separable Hilbert spaces $\mathscr{H}_{v}$, one for each $v \in V(\Gamma)$;

- a collection of isometries $U_{\vec{e}}: \mathscr{H}_{s(\vec{e})} \rightarrow \mathscr{H}_{t(\vec{e})}$ for each oriented edge $\vec{e}$ such that $U_{\vec{e}} U_{\vec{e}^{\prime}}=1$ whenever $\vec{e}$ and $\overrightarrow{e^{\prime}}$ are the two orientations of the same edge. 
Remark 2.4. This construction can also be stated in more categorical terms. It is a certain quiver representation. A finite graph $\Gamma$ also generates a groupoid (that is a category in which all morphisms are invertible) and in this setting a representation is a functor from the groupoid to the category of separable Hilbert spaces.

Definition 2.5. Let $\rho_{\Gamma}$ be a representation of a finite graph $\bar{\Gamma}$ and write $\mathscr{H}_{\bar{\Gamma}}:=$ $\bigoplus_{v \in V(\bar{\Gamma})} \mathscr{H}_{v}$. Define the graph Harper operator $H$ on $\mathscr{H}_{\bar{\Gamma}}$ by

$$
H:=\sum_{\text {oriented edges } \vec{e}} U_{\vec{e}},
$$

where we have (ab)used the notation $U_{\vec{e}}$ to denote the partial isometry on $\mathscr{H}_{\vec{\Gamma}}$ induced by the operator of the same name.

\subsection{Matrix actions}

2.3.1. General setup. Fix some finite index set $I$, an index $o \in I$, and order on $I$ such that $o$ is the smallest element. Fix isomorphic Hilbert spaces $\mathscr{H}_{i}, i \in I$ and let $\phi_{i}: \mathscr{H}_{o} \rightarrow \mathscr{H}_{i}$ be fixed choice of isomorphisms. We allow a choice of $\phi_{o}$. It might be that $\phi_{o}=$ id but this is not necessary.

Set $\mathscr{H}=\bigoplus_{i \in I} \mathscr{H}_{i}$.

In this situation there is an action of $M_{|I|}\left(\operatorname{End}\left(\mathscr{H}_{o}\right)\right)$ on $\mathscr{H}$ given as follows. Let $A \in M_{|I|}\left(\operatorname{End}\left(\mathscr{H}_{o}\right)\right)$ be an endomorphism valued matrix. Then the action of $A$ on $\mathcal{H}$ is given by

$$
\mathscr{H}=\bigoplus_{i \in I} \mathscr{H}_{i} \stackrel{\oplus_{i} \phi_{i}^{-1}}{\longrightarrow} \mathscr{H}_{o}^{\oplus|I|} \stackrel{A}{\longrightarrow} \mathscr{H}_{o}^{\oplus|I|} \stackrel{\oplus_{i} \phi_{I}}{\longrightarrow} \mathscr{H} .
$$

Vice versa, given an endomorphism $H \in \operatorname{End}(\mathscr{H})$ there is a corresponding matrix $M(H) \in M_{|I|}\left(\mathscr{H}_{o}\right)$ given by

$$
\mathscr{H}_{o}^{\oplus|I|} \stackrel{\oplus_{i} \phi_{i}}{\longrightarrow} \mathcal{H} \stackrel{H}{\longrightarrow} \mathcal{H} \stackrel{\oplus_{i} \phi_{i}^{-1}}{\longrightarrow} \mathcal{H}_{o}^{\oplus|I|} .
$$

That is, if $\Phi=\bigoplus_{i} \phi_{i}$, then $M(H)=\Phi^{-1} \circ H \circ \Phi$.

2.3.2. Matrix action for the graph Harper operator. In order to write the Harper operator of $\S 2.2$ as a matrix according to $\$ 2.3 .1$ we need to fix several choices. We will assume that the graph $\Gamma$ is connected. First there is a choice of base vertex $v_{o}$ and a choice of order on all vertices in which the base vertex is the smallest. Then for each vertex $v$ we choose a fixed path $p_{v}$ - that is, a sequence of ordered edges $\vec{e}_{1}, \ldots, \vec{e}_{k}$ - from $v_{o}$ to $v$. We then set $\phi_{v}:=\phi_{\vec{e}_{k}} \circ \cdots \circ \phi_{\vec{e}_{1}}: \mathscr{H}_{v_{o}} \rightarrow \mathscr{H}_{v}$. If $v=v_{o}$ we also allow the choice $\phi_{v_{o}}:=$ id: $\mathscr{H}_{v_{o}} \rightarrow \mathscr{H}_{v_{o}}$. This corresponds to the empty path. 
Given such a choice, we obtain the action $\rho_{p_{1}, \ldots, p_{n}}$ as above. We call the resulting matrix the graph Harper operator in matrix form.

Given a rooted spanning tree $\tau$ of $\Gamma$, choose $v_{0}$ to be the root, and then there is a unique shortest path on $\tau$ from $v_{0}$ to any vertex $v_{i}$ of $\tau$, which we can view as a path on $\Gamma$. Thus we get the data needed. Again a convenient choice of paths is given by a spanning tree. We sometimes write $H_{\Gamma, \tau}$ for the corresponding graph Harper operator in matrix form.

Remark 2.6. Any two matrix Harper operators are conjugate. This follows simply by making a base change.

\section{3. $C^{*}$-geometry of Harper Hamiltonians on lattices}

For lattices there are different definitions and connotations in the mathematics and physics literature. We will use the adjectives "mathematical" and "physical" to distinguish the two. As a reference in the physics literature for the definition of lattices we use [1].

3.1. Translation action. The additive Abelian group $\mathbb{R}^{n}$ acts on itself by translation. Write $T_{w}(v)=v+w$. For a subset $S \in \mathbb{R}^{n}$ and a subgroup $L \subset \mathbb{R}^{n}$ we denote by $L(S)$ the set of all translates of points in $S$ under the action of all elements of $L$.

\subsection{Lattices: mathematical, physical and Bravais}

Definition 3.1. A mathematical lattice with a basis of rank $m$ in $\mathbb{R}^{n}$ is an injective group homomorphism $\rho_{L}: \mathbb{Z}^{m} \hookrightarrow \mathbb{R}^{n}$. If $m=n$ then such a lattice is called a Bravais lattice with a basis.

If $\rho$ is a mathematical lattice with a basis, we let $L:=\rho_{L}\left(\mathbb{Z}^{m}\right)$ be its image. This is a discrete subgroup of $\mathbb{R}^{n}$, which is isomorphic to $\mathbb{Z}^{n}$. We will refer to $L$ as a mathematical/Bravais lattice.

A Bravais subgroup or sublattice is a subgroup $L^{\prime} \subset L$ which is a Bravais lattice. Any Bravais lattice $L$ acts by translations on $\mathbb{R}^{n}$ and a primitive cell is a fundamental domain for this action. In general a cell is a fundamental domain for a Bravais subgroup $L^{\prime}$ of $L$.

Example 3.2. The integer points $\mathbb{Z}^{n} \subset \mathbb{R}^{n}$ are a Bravais lattice, which we call the crystallographic lattice. A crystallographic cell is a primitive cell for this lattice. The unit cube is a primitive cell. The cube of length 2 is a cell that is not primitive.

As a definition for a lattice in the physical sense, we will take the following convention: 
Definition 3.3. A subset $\Lambda \subset \mathbb{R}^{n}$ is a lattice if there is a finite set $V \subset \mathbb{R}^{n}$ and a Bravais lattice $L$ such that $\Lambda=L(V)$.

The tuple $(L, V)$ is called a crystal structure or a lattice with a basis. ${ }^{5}$ We will not use the latter terminology as it is mathematically confusing.

Notice that given $\Lambda$ neither $L$ nor $V$ are uniquely defined. We can and will mostly choose $L$ to be maximal and $V$ to be minimal or primitive. In this case we call $(L, V)$ a primitive crystal structure. Sometimes $L$ is called the space group.

Still there is of course a choice in fundamental domain and hence a choice in $V$, but for any two such choices there is a unique bijection. In fact if we let $\bar{V}$ be a set of orbits of the $L$ action; then it is unique and any $V$ is just a choice of representatives. It is also easy to see when $L$ is minimal. $L$ is minimal if and only if the number of orbits $|\bar{V}|$ is minimal.

3.3. Graphs and lattices. To develop a general theory, we will deal with two cases. The usual case is that given a lattice we obtain a graph by adding edges to the nearest neighbors. But is convenient to also allow that we already have an embedded graph $\Gamma_{\Lambda}$ whose vertices are the set $\Lambda$. In this case, we will let $\Gamma_{\Lambda}$ stand for this chosen graph.

3.3.1. Canonical graph of a lattice. Given a lattice $\Lambda$ without a pre-chosen graph define the graph $\Gamma_{\Lambda}$ of $\Lambda$ to be the graph whose vertices are the elements of $\Lambda$ and whose edges are the line segments between nearest neighbors. This graph is naturally embedded in $\mathbb{R}^{n}$ and we will sometimes make use of this.

3.3.2. Quotient graphs. Given a choice of symmetry group $L$ for $\Lambda$, we also define the quotient graph $\bar{\Gamma}_{\Lambda}(L)$ as $\Gamma_{\Lambda} / L \subset \mathbb{R}^{n} / L$. This is the abstract graph whose vertices are given by $\bar{V}$ and whose edges are given by the set of orbits of edges of $\Gamma_{\Lambda}$.

If $L$ is a maximal group we will just write $\bar{\Gamma}_{\Lambda}$.

If the unit cell is a cell for $L$ corresponding to a subgroup $L^{\prime}$, we also define the crystallographic quotient graph of $\Lambda$ to be the graph $\bar{\Gamma}_{\Lambda}\left(L^{\prime}\right)$.

3.4. Group of a lattice. A lattice $\Lambda$ also generates a discrete subgroup of $\mathbb{R}^{n}$ as follows: using the natural embedding $\Gamma_{\Lambda} \subset \mathbb{R}^{n}$ we can think of any directed edge $\vec{e}$ of $\Gamma_{\Lambda}$ of $\Gamma_{\Lambda}$ simply as a vector in $\mathbb{R}^{n}$. Then there is an associated translation operator $T_{\vec{e}}$. Notice that as vectors the translates of all the oriented edges correspond to one another. This means that the set of vectors $\vec{e}$ is given by one vector in each orbit of oriented edges of the embedded graph $\Gamma_{\Lambda}$. This set is in bijection with the oriented edges of $\bar{\Gamma}_{\Lambda}$ and we will not distinguish between them in the notation. That is, we write $\vec{e}$ for both the oriented edge of the abstract graph $\bar{\Gamma}_{\Lambda}$ and the vector in $\mathbb{R}^{n}$ it is

\footnotetext{
${ }^{5}$ Usually one would include labels such as atom labels to $V$.
} 
in bijection with. We will enumerate the set of vectors $\{\vec{e}\}$ as $\vec{e}_{i}$. These vectors then give a symmetric group of generators of an Abelian subgroup of $\mathbb{R}^{n}$, which we call the lattice group $T(\Lambda)$ of $\Lambda$. It is the group generated by the $\vec{e}_{i}$ This group consists of all elements of the form

$$
T(\Lambda):=\left\{\sum_{i} a_{i} \vec{e}_{i} \mid a_{i} \in \mathbb{Z}\right\}
$$

Proposition 3.4. $T(\Lambda)$ is a mathematical lattice.

Proof. To generate the group we only need one choice of orientation per edge. Let $\vec{e}_{j}, j \in J$, be such a choice. This choice defines a map $\mathbb{Z}^{J} \rightarrow \mathbb{R}^{n}$. The image of this group is a torsion-free Abelian group and hence by the structure theorem for Abelian groups a free Abelian group of rank $\leq|J|$.

$T(\Lambda)$ also naturally acts by translations on $\mathbb{R}^{n}$.

3.5. Fundamental group. The graph $\Gamma_{\Lambda}$ is a covering space for $\bar{\Gamma}_{\Lambda}$. Since we know the fundamental group of $\bar{\Gamma}_{\Lambda}$ by Proposition (2.1), this information can be used to calculate the fundamental group of $\Gamma_{\Lambda}$. In order to do the computation, we will have to fix some notation. We fix a rooted spanning tree $\tau$ of $\bar{\Gamma}_{\Lambda}$ and set $n:=\left|E\left(\bar{\Gamma}_{\Lambda}\right)\right|-|E(\tau)|$. By contracting $\tau$ we obtain a surjection $\pi: \bar{\Gamma}_{\Lambda} \rightarrow \wedge_{i=1}^{n} S^{1}$, which by Proposition (2.1) induces an isomorphism on fundamental groups. We fix generators of $\pi_{1}\left(\wedge_{1}^{n} S^{1}\right)$ and choose lifts $l_{1}, \ldots, l_{n}$ of them. By definition each loop $l_{i}$ is a sequence of directed edges $\vec{e}_{i_{1}}, \ldots, \vec{e}_{i_{k(i)}}$ for some $k(i)$. We set $\vec{l}_{i}:=$ $\vec{e}_{i_{1}}+\cdots+\vec{e}_{i_{k(i)}}$.

Proposition 3.5. Let $\Lambda$ be a lattice with graph $\Gamma_{\Lambda}$ and finite quotient graph $\bar{\Gamma}_{\Lambda}$. Let $\tau$ be a rooted spanning tree for $\bar{\Gamma}_{\Lambda}$ and set $n:=\left|E\left(\bar{\Gamma}_{\Lambda}\right)\right|-|E(\tau)|$. If the vectors $\vec{l}_{i}$ are linearly independent, then $\pi_{1}\left(\Gamma_{\Lambda}\right)=\left[\mathbb{F}_{n}, \mathbb{F}_{n}\right]$. Furthermore a loop on $\bar{\Gamma}_{\Lambda}$ lifts to a loop on $\Gamma_{\Lambda}$ if and only if it traverses each edge the same number of times in each direction.

Proof. We need to compute which loops on $\bar{\Gamma}_{\Lambda}$ lift to loops on $\Gamma_{\Lambda}$ and which do not. Given a loop $l$ on $\bar{\Gamma}_{\Lambda}$ it can be written as a word in the basis loops $l_{i}$, say $w=\prod_{j} l_{i_{j}}^{\epsilon(j)}$ where $\epsilon(j)= \pm 1$. Fixing a pre-image of $v_{0}$ given by the rooted spanning tree, this lifts to an edge path on $\Gamma_{\Lambda}$ given by the sequences of vectors $\vec{e}_{i_{1}}, \ldots, \vec{e}_{i_{k(i)}}$. Notice that $l_{i}^{-1}$ gives rise to the sequence $-\vec{e}_{i_{k(i)}}, \ldots,-\vec{e}_{i_{1}}$. These vectors form a closed loop if and only if they all sum up to $\overrightarrow{0}$. Now each $l_{i}^{ \pm 1}$ contributes $\pm \vec{l}_{i}$ to the sum. This means that $w$ lifts to a loop if and only if $\sum_{j} \epsilon(j) \vec{l}_{i}=\overrightarrow{0}$. Since by assumption the $\vec{l}_{i}$ are linearly independent, this happens if and only if for fixed $i$ the sum of the exponents of the occurring $l_{i}$ is zero, and this happens precisely if the image of $w$ in the Abelianization $\operatorname{Ab}\left(\mathbb{F}_{n}\right):=\mathbb{F}_{n} /\left[\mathbb{F}_{n}, \mathbb{F}_{n}\right]=\mathbb{Z}^{n}$ is 0 . This means that the covering 
group of the cover $\Gamma_{\Lambda} \rightarrow \bar{\Gamma}_{\Lambda}$ is $\mathrm{Ab}\left(\mathbb{F}_{n}\right)$, which is a normal subgroup of $\mathbb{F}_{n}$ and hence the covering group of $\Gamma_{\Lambda}$ is $\left[\mathbb{F}_{n}, \mathbb{F}_{n}\right]$. The last statement follows immediately by noticing that this is true for the $\vec{l}_{i}$ and hence also for their summands $\overrightarrow{e_{j}}$.

\subsection{Main examples}

3.6.1. The honeycomb lattice. The honeycomb lattice (see Figure 7) is an example of a physical lattice. To make things precise, we let $\Lambda_{\text {hex }} \subset R^{2}$, which is the Bravais lattice generated by $-e_{1}:=(1,0)$ and $e_{3}:=\frac{1}{2}(1,-\sqrt{3})$. We set $e_{2}=-e_{1}-e_{3}=$ $\frac{1}{2}(1, \sqrt{3})$. A "dual" Bravais lattice $\Lambda_{\text {hex }}^{\mathrm{t}}$ generated by $f_{2}:=e_{2}-e_{1}=\frac{1}{2}(-3, \sqrt{3})$ and $f_{3}:=e_{3}-e_{1}=\frac{1}{2}(3, \sqrt{3})$ acts via translation on $\Lambda_{\text {hex }}$. There are precisely three orbits of this action. These are the $A, B$ and $C$ lattices, where we fix the $A$ lattice to be the orbit of $(1,0)$, the $B$ lattice to be the orbit of $(-1,0)$, and the $C$ lattice to be the orbit of $(0,0)$. The lattice $\Lambda_{\mathrm{hc}}$ is then defined to be the union of the $A$ and $B$ sublattices. In $\Lambda_{\mathrm{hc}}$ there are three nearest neighbors as indicated in Figure 7.

The symmetry group is $\mathbb{Z}^{2}$ embedded as the "dual" Bravais lattice above. The group $T\left(\Lambda_{\mathrm{hc}}\right)$ is again isomorphic to $\mathbb{Z}^{2}$, but it is the original triangular lattice generated by $e_{1}$ and $e_{3}$. See Figure 7 . This defines the graph $\Gamma_{\Lambda_{\mathrm{hc}}}$.

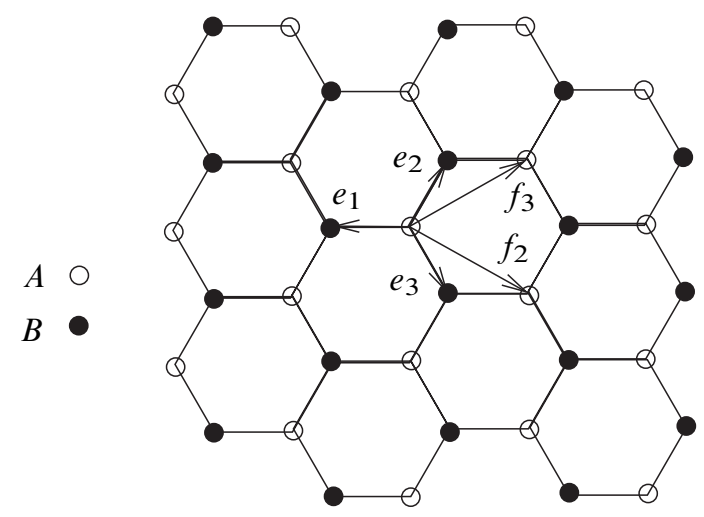

Figure 7. The honeycomb lattice $\Lambda_{\text {hon }}$, the generators $f_{i}$ for $L\left(\Lambda_{\text {hon }}\right)$ and the generators $e_{i}$ of $T\left(\Lambda_{\text {hon }}\right)$.

3.6.2. The gyroid lattice graph. The gyroid lattice is given by the set of vertices $V_{+}$of $\Gamma_{+}$of $\S 1$. It is also a physical lattice. Here the symmetry group is the space group of $I 4_{1} 32$, which is the body centered cubic (bcc) lattice. The group $T(\Lambda)$ is actually the face centered cubic (fcc) lattice as shown in Proposition 1.8.

3.7. Hilbert space of a lattice. Given a lattice or in general a countable set $\Lambda$, we define its Hilbert space to be $\mathscr{H}_{\Lambda}:=l^{2}(\Lambda)$, which is the set of square summable 
complex sequences indexed by $\Lambda$. There is an alternative way to think about a sequence $\left(a_{\lambda}: \lambda \in \Lambda\right)$ as a function $\psi$ on $\Lambda$. Given a sequence as above, the function is given by $f(\lambda):=a_{\lambda}$. Vice versa, given $\psi$ the sequence is obtained by setting $a_{\lambda}:=\psi(\lambda)$.

Given an action of a group $G$ on $\Lambda$ there is an induced action of $G$ on $l^{2}(\Lambda)$ given by $g \psi(\lambda):=\psi\left(g^{-1}(\lambda)\right)$ for $g \in G$ and $\psi \in l^{2}(\Lambda)$.

A standard basis for $l^{2}(\Lambda)$ is given by the functions $v_{l}\left(l^{\prime}\right)=\delta_{l, l^{\prime}}$.

If $\Lambda$ is a lattice then there is a unitary representation of $\Lambda$ on $\mathscr{H}=l^{2}(\Lambda)$, given via the translation operators $T_{l}\left(v_{l^{\prime}}\right)=v_{l+l^{\prime}}$.

Given a lattice $\Lambda$ and fixing a translation group $L$, we can decompose the Hilbert space $\mathscr{H}_{\Lambda}$ by breaking it down in terms of the orbits of $L$. More precisely, labeling each orbit by a vertex in $\bar{\Gamma}$, we obtain the direct sum decomposition

$$
\mathscr{H}_{\Lambda}=\bigoplus_{\bar{v} \in \bar{\Gamma}} \mathscr{H}_{\bar{v}}
$$

where $\mathscr{H}_{v}=l^{2}(L(v))$ for any $v$ which represents the class of $\bar{v}$.

3.8. Partial isometries and projections. In general we get a representation of $L(\Lambda)$ on $\mathscr{H}_{\Lambda}$. Notice that there is in general no representation of $T(\Lambda)$ on $\mathscr{H}_{\Lambda}$. We do have a partial action, which is given by partial isometries. If $\vec{e}$ is an edge from $v$ to $w$ in $\bar{\Gamma}$, then it induces an isomorphism $V_{\vec{e}}: \mathscr{H}_{w} \rightarrow \mathscr{H}_{v}$ simply by setting $V_{\vec{e}} \psi(l)=\psi(l-\vec{e})$. This induces a partial isometry on all of $\mathscr{H}(\Lambda)$.

Another way to describe this partial isometry is as follows. Consider $\mathscr{H}_{\Lambda}$. Since $T(\Lambda) \supset \Lambda$, after shifting $\Lambda$ so that $0 \in \Lambda$, we have $\mathscr{H}_{T(\Lambda)} \supset \mathscr{H}_{\Lambda}$, and moreover $T(\Lambda)$ acts on $\mathscr{H}_{T(\Lambda)}$ via translation operators $T_{\vec{e}}$. If $P$ is the orthogonal projection of $\mathscr{H}_{T(\Lambda)}$ to $\mathscr{H}_{\Lambda}$ then $V_{\vec{e}}=P T_{\vec{e}} P$. If furthermore $P_{v}$ is the projection onto $\mathscr{H}_{v} \subset$ $H_{T(L)}$, then we can further decompose the action into components $U_{\vec{e}}^{w, v}=P_{v} T_{\vec{e}} P_{w}$. Notice that in principle there could be a directed edge $e^{\prime} \neq e$ with $\overrightarrow{e^{\prime}}=\vec{e}$ as vectors in $\mathbb{R}^{n}$. This happens for e.g. the cube in $\$ 1.3 .1$. In this case there will be more components or super selection sectors to use physics terminology.

3.9. Projective representations. Up to now we have insisted that the translation operators are a bona fide representation of the translations groups.

It turns out that to accommodate such physical data as a magnetic field one should only expect a projective representation. This is also in accord with general quantum theory, where representations are always only expected to be projective.

This is also one of the sources of noncommutativity, the second being that one does not expect the symmetries of the system to commute with the Hamiltonian on the nose. One actually has a choice either to preserve the commutativity of the symmetry operators or to preserve that the symmetry operators commute with the Hamiltonian. We recall some standard facts from representation theory. 
3.9.1. Cocycles. A $U(1) 2$-cocycle for a group $G$ written as $c \in Z^{2}(G, U(1))$ is a map $c: G \times G \rightarrow U(1)$ such that

$$
c(u, v) c(u v, w)=c(u, v w) c(v, w),
$$

which is called a cocycle condition.

Notice that if $c$ is a $U(1) 2$-cocycle for $G$ and $H \subset G$ is a subgroup, then its restriction $\left.c\right|_{H}: H \times H \rightarrow U(1)$ is a $U(1)$ 2-cocycle for $H$.

Definition 3.6. A morphism $\rho: G \rightarrow U(\mathscr{H})$ is called a projective representation with cocycle $c$ if $\rho\left(1_{G}\right)=\mathrm{id}_{\mathscr{H}}$, that is, the identity $1_{G}$ of $G$ maps to the identity operator id $\mathscr{H}$ and $\rho(u) \rho(v)=c(u, v) \rho(u+v)$.

Example 3.7. A cocycle $c$ is called trivial if $c(u, v)=s(u) s(v) s^{-1}(u v)$ for some group morphism $s: G \rightarrow U(1)$. Given a trivial group cocycle one can always perturb or scale an existing representation $\rho$ to a projective representation $\rho_{c}$ with cocycle $c$ by setting $\rho_{c}(u):=s(u) \rho(u)$. With the same formula one can scale a projective representation with a cocycle $c^{\prime}$ to one with the cocycle $c c^{\prime}$.

Remark 3.8. We will be dealing with Abelian groups $G$, viz. $\mathbb{Z}^{n}$, for which we will use the usual additive notation of 0 and + for the identity and group operation.

In the case of a free Abelian group and its Hilbert space, given any cocycle, one can also twist the standard representation to a projective representation with that cocycle.

Lemma 3.9. Let $L \simeq \mathbb{Z}^{n} \subset \mathbb{R}^{n}$ be a lattice and $\alpha \in Z^{2}(L, U(1))$. Then the operators $U_{l}$ which operate on $\mathscr{H}_{L}$ via

$$
U_{l}\left(v_{l^{\prime}}\right)=\alpha\left(l, l^{\prime}\right) v_{l+l^{\prime}}
$$

where $v_{l}$ is the standard basis, satisfy

$$
U_{l} U_{l^{\prime}}=\alpha\left(l, l^{\prime}\right) U_{l+l^{\prime}}, \quad U_{l} U_{l^{\prime}}=\epsilon\left(l, l^{\prime}\right) U_{l^{\prime}} U_{l} \quad \text { with } \epsilon\left(l, l^{\prime}\right)=\frac{\alpha\left(l, l^{\prime}\right)}{\alpha\left(l^{\prime}, l\right)} .
$$

Proof. Straightforward calculation.

Lemma 3.10. Let $B$ be a bilinear form on $\mathbb{R}^{n}$. Then $\alpha_{B}(u, v):=e^{\frac{i}{2} B(u, v)}$ is a 2-cocycle for $\mathbb{R}^{n}$. If $\Lambda \subset \mathbb{R}^{n}$ is a lattice and $e_{i}$ are generators for this lattice, then the algebra of operators $U_{l}$ of the $\alpha$ twisted representation is generated by the operators $U_{i}:=U_{e_{i}}$.

Proof. First we calculate

$$
\begin{aligned}
\alpha_{B}(u, v) \alpha_{B}(u+v, w) & =\exp \left(\frac{i}{2}[B(u, v)+B(u+v, w)]\right) \\
& =\exp \left(\frac{i}{2}[B(u, v)+B(u, w)+B(v, w)]\right), \\
\alpha_{B}(u, v+w) \alpha_{B}(v, w) & =\exp \left(\frac{i}{2}[B(u, v+w)+B(v, w)]\right) \\
& =\exp \left(\frac{i}{2}[B(u, v)+B(u, w)+B(v, w)]\right) .
\end{aligned}
$$


Secondly, if $l=\sum a_{i} e_{i}$, then $\prod U_{i}^{a_{i}} \propto U_{l}$ by the formulas (7).

3.9.2. Noncommutative tori. A standard example which will be important in the following is given by the projective action of $\mathbb{Z}^{n}$ on $\mathscr{H}\left(\mathbb{Z}^{n}\right)$ with the cocycle $\alpha$ given by choosing an anti-symmetric bilinear form $B=2 \pi \widehat{\Theta}$ on $\mathbb{R}^{n}$ and then restricting the cocycle. This yields a cocycle which we call $\alpha_{\widehat{\Theta}}:=\alpha_{B}(u, v)=e^{i \pi \hat{\Theta}(u, v)}$.

In this case the operators $U_{i}:=U_{e_{i}}$ generate the algebra of operators $U_{l}$. These generators satisfy

$$
U_{i} U_{j}=e^{2 \pi i \Theta_{i j}} T_{j} T_{i}, \quad \Theta_{i j}=\widehat{\Theta}\left(e_{i}, e_{j}\right)
$$

This follows from (7); $\epsilon\left(U_{i}, U_{j}\right)=e^{i \pi \Theta_{i j}} e^{-i \pi \Theta_{j i}}=e^{2 \pi i \Theta_{i j}}$ by anti-symmetry of $\Theta$. In general:

Definition 3.11. For a fixed anti-symmetric $(n \times n)$-matrix $\Theta$ the $\mathrm{C}^{*}$-algebra generated by $n$ unitary operators $U_{i}$ on a separable Hilbert space satisfying the commutation relations (8) is called a noncommutative torus and denoted by $\mathbb{T}_{\Theta}^{n}$.

Note that $\mathbb{T}^{n}:=\mathbb{T}_{0}^{n}$ is the commutative $\mathrm{C}^{*}$-algebra corresponding to the torus $T^{n}=\left(S^{1}\right)^{\times n}$ under the Gelfand-Naimark theorem.

Example 3.12. $n=2$ : In this case the skew-symmetric matrix can be written as $\Theta=\theta\left(\begin{array}{cc}0 & 1 \\ -1 & 0\end{array}\right)$. And $\alpha\left(l, l^{\prime}\right)=\exp \left[i \pi \theta\left(l \wedge l^{\prime}\right)\right]$, where for $l=\left(l_{1}, l_{2}\right)$ and $l^{\prime}=\left(l_{1}^{\prime}, l_{2}^{\prime}\right): l \wedge l^{\prime}:=\operatorname{det}\left(\begin{array}{ll}l_{1} & l_{1}^{\prime} \\ l_{2} & l_{2}^{\prime}\end{array}\right)$ and accordingly $\epsilon\left(l, l^{\prime}\right)=e^{2 \pi \theta l \wedge l^{\prime}}$. This case is written as $\mathbb{T}_{\theta}^{2}$.

Remark 3.13. As usual, once a basis $b_{i}$ for $\mathbb{R}^{n}$ is fixed, there is a bijection between anti-symmetric bilinear forms $\widehat{\Theta}$ and skew-symmetric matrices $\Theta$ given by $\Theta_{i j}=$ $\widehat{\Theta}\left(b_{i}, b_{j}\right)$. If thus choice of basis has been made, we will write $\alpha_{\Theta}$. In our applications, the basis $b_{i}$ will be given to us by a choice of basis for the Bravais lattice $L$.

3.9.3. Wannier or magnetic translation operators. In case of magnetic field there is a standard cocycle and representation coming from the $B$-field. This was first used in [12]. A magnetic field $B$ in mathematical terms is a 2 -form on $\mathbb{R}^{n}$.

If we assume that the $B$-field is constant, then this is nothing but a skew-symmetric bilinear form on $\mathbb{R}^{n}$, thus giving rise to a cocycle $\alpha_{B}$ as above. This cocycle can now be restricted to any lattice in $\mathbb{R}^{n}$. Furthermore in this situation, we can choose a magnetic potential $A$. This is a 1 -form on $\mathbb{R}^{n}$ such that $d A=B$. This form exists since $d B=0$, and as $\mathbb{R}^{n}$ is contractible, $\bar{H}^{*}\left(\mathbb{R}^{n}\right)=0$, i.e., the reduced cohomology vanishes so that every closed form is exact.

In this case the cocyle is trivial and the twisted action can be rewritten as

$$
U_{l^{\prime}} \psi(l)=e^{-i \int_{l}^{\left(l-l^{\prime}\right)} A} \psi\left(l-l^{\prime}\right) .
$$


Remark 3.14. If $\sum_{i=1}^{n} m_{i}=0$ is a closed cycle of vectors bounding a simply connected polygonal region $D$ with vertices $v_{1}, \ldots, v_{n}$ such that $v_{i+1}=v_{i}+m_{i}$, then $U_{m_{1}} \ldots U_{m_{n}}=e^{-i F}$ id, where $F$ is the flux of $B$ through $D$. Furthermore, if $B$ has a nonsingular vector potential $A$ such that $\operatorname{curl}(A)=B$, then $F=\int_{D} B d S=$ $\sum_{i} \int_{0}^{1} m_{i} \cdot A\left(v_{i}+m_{i} t\right) d t$.

This means that given two elements $l, l^{\prime}$ we have

$$
U_{l} U_{l^{\prime}}=\epsilon\left(l, l^{\prime}\right) U_{l^{\prime}} U_{l}, \quad \epsilon\left(l, l^{\prime}\right)=e^{i \int_{R} B d S},
$$

where $R$ is the rectangle spanned by the vectors $l$ and $l^{\prime}$.

Example 3.15. In the square lattice, if we choose a constant magnetic field in $z$ direction $\vec{B}=\vec{k}$ or $B=2 \pi \theta d x \wedge d y$, then we can choose $A=\frac{1}{2}\left(\alpha_{1} y d x+\alpha_{2} x d y\right)$ with $2 \pi \theta=\alpha_{2}-\alpha_{1}$. Setting $U:=U_{e_{1}}$ and $V=U_{e_{2}}$ we obtain $U V=e^{2 \pi i \theta} V U$ and this is just $e^{i \phi}$ where $\phi=\int_{D} B d A$ is the flux through the domain full square $A$ spanned by $e_{1}$ and $e_{2}$. The resulting algebra is $\mathbb{T}_{\theta}^{2}$.

3.10. Harper operator for a lattice $\Lambda$ and with graph $\Gamma_{\Lambda}$. In this section we construct a Harper operator for a given lattice $\Lambda$ and a graph for the lattice $\Gamma_{\Lambda}$. Associated to each such lattice there is a natural separable Hilbert space $\mathscr{H}_{\Lambda}$. The Harper operator is an operator on $\mathscr{H}_{\Lambda}$ which is obtained by giving a graph representation of $\bar{\Gamma}$. To do this we fix a maximal translational symmetry group $L$ for $\Lambda$ (see §3.2). Using the techniques of $\$ 2$ we obtain a Harper operator as we discuss in detail below. This operator together with the representation of the translation group $L$ defines a subalgebra of the endomorphism algebra of $\mathscr{H}_{\Lambda}$, which we call the Bellissard-Harper algebra and denote by $\mathscr{B}\left(\Gamma_{\Lambda}, L, \alpha\right)$ or $\mathscr{B}$ for short. Here we allow for projective representations acting with the cocycle $\alpha$, e.g. via a magnetic field (see §3.9.3). If $\Gamma_{L}, L$ and $\alpha=\alpha_{B}$ with $B=2 \pi i \widehat{\Theta}$ is fixed, we write $\mathscr{B}_{\widehat{\Theta}}$ for the corresponding Bellissard-Harper algebra.

Furthermore again using $\S 2$, we can get a a matrix representation for the Harper operator and the action of $L$. These matrices are elements of a matrix algebra with coefficients in the algebra generated by the operators corresponding to $L$. Fixing $\Gamma_{\Lambda}$, $L$, a path basis, a basis for $L$ and a magnetic field $B=2 \pi \hat{\Theta}$, we arrive at an algebra of matrices, which we call $\mathscr{B}_{\Theta}$. The details are given below.

3.10.1. Graph representation of $\overline{\boldsymbol{\Gamma}}$. To put ourselves in the situation of $\S 2.3 .1$, we will decompose $\mathscr{H}_{\Lambda}$ as

$$
\mathscr{H}_{\Lambda}=\bigoplus_{\bar{v} \in \bar{\Gamma}} \mathcal{H}_{\bar{v}}
$$

where $\mathscr{H}_{v}=l^{2}(L(v))$ for any $v$ which represents the class of $\bar{v}$.

We also fix a 2-cocycle $\alpha_{B}$ corresponding to a skew-symmetric bilinear form or, equivalently, a constant $B$-field. This gives us the Hilbert space part of the data of a 
graph representation $\rho_{\bar{\Gamma}}$. We define the isomorphisms as $U_{\vec{e}}$. For this we use the fact that an oriented edge of $\bar{\Gamma}$ has a natural representation as a vector $\vec{e}$ (see 3.4 ).

Definition 3.16. The Harper operator $H_{\Lambda}$ is defined to be the graph Harper operator $H_{\bar{\Gamma}}$ on $\mathscr{H}_{\Lambda}$ corresponding to the graph representation $\rho_{\Gamma}$.

The Bellissard-Harper $(\mathrm{BH})$-algebra $\mathcal{B}\left(\Gamma_{\Lambda}, L, \alpha_{B}\right)$ is the $\mathrm{C}^{*}$-algebra of operators on $\mathscr{H}_{\Lambda}$ generated by the projective representation of $L$ and the graph Harper operator.

Remark 3.17. The operator $\mathscr{H}_{\Lambda}$ also generates random walks and is related to a discrete difference operator as follows. Let $\Gamma_{\Lambda}$ be a $k$-regular graph, which means that each vertex has valence $k$. Then $\Delta=k-H_{\Lambda}$ acts as the difference operator. We have $\Delta(\Psi)(l)=\left(\sum_{\vec{e}: s(\vec{e})=l} \Psi(l)-\Psi\left(T_{\vec{e}}(l)\right)\right)$, where the sum is over "nearest neighbors" as defined by $\Gamma_{\Lambda}$.

3.10.2. A matrix representation of the Bellissard-Harper algebra. In order to get a matrix representation, we fix a vertex $v_{o}$ of $\bar{\Gamma}_{\Lambda}$ and a choice of paths $p_{v}$ from $v_{o}$ to $v$. We will call such a choice a choice of path basis. Again a convenient way to fix such data is to specify a spanning tree.

We then get a matrix representation of the Harper operator and the operators coming from the projective representation of $L$.

Theorem 3.18. Fixing a choice of path basis and a basis for $L$, the corresponding faithful matrix representation of $\mathcal{B}\left(\Gamma_{\Lambda}, L, \alpha_{B}\right)$ is a sub-C*-algebra $\mathcal{B}_{\Theta}$ of the $C *_{-}$ algebra $M_{|V(\bar{\Gamma})|}\left(\mathbb{T}_{\Theta}^{n}\right)$.

Proof. Before passing to the matrix representation, all the operators involved are shifted translation operators, those coming from $L$ and those coming from $L(\Lambda)$. First we have to show that the operators from $L$ still act as operators from $L$ when restricted to $\mathscr{H}_{v_{o}}$, but this is clear since they are diagonal in the direct sum decomposition (6). Thus the operators in question are conjugates, $U_{p_{v}} U_{l} U_{p_{v}}^{*} \propto U_{l}$ for any $U_{l} \in \mathbb{T}_{\Theta}^{n}$. Here $\Theta$ is the matrix obtained from $\widehat{\Theta}=\frac{1}{2 \pi} B$ by using the choice of basis of $L$. Secondly, for $l^{\prime} \in T(\Lambda), U_{p_{v}} U_{l^{\prime}} U_{p_{v}^{\prime}}^{*} \propto U_{l^{\prime \prime}}$ acts as a translation operator which preserves the $v_{o}$ summand. This means that the sum of vectors $l^{\prime \prime}=-p_{v}+l^{\prime}-p_{v}^{\prime}$ is actually in $L$. Hence the assertion follows.

Notice that different choices of path basis may lead to different representations, but all these representations are isomorphic; moreover they are conjugates of one another. The effect of changing the basis of $L$ is to replace the matrix $\Theta$ with its basis transform $\Theta^{\prime}$, but as $\mathrm{C}^{*}$-algebras $\mathbb{\Psi}_{\Theta}^{n}=\mathbb{\mathbb { Q }}_{\Theta^{\prime}}^{n}$ - only the presentation has changed with the base change acting as an endomorphism.

Corollary 3.19. If $\Theta$ is rational then the spectrum of $H_{\Lambda}$ has finitely many gaps. Moreover, the maximal number is determined by the entries of $\Theta$. 
Proof. Since there is an injection of $\mathscr{B}_{\Theta}$ into $M_{|V(\bar{\Gamma})|}\left(\mathbb{T}_{\Theta}^{n}\right)$, we can restrict the tracial states to $\mathscr{B}_{\Theta}$. The image of the tracial states of $\mathbb{\mathbb { T }}_{\Theta}^{n}$ is known to be $S=\mathbb{Z}+$ $\sum_{i j} \theta_{i j} \mathbb{Z} \subset \mathbb{R}$ [6], [7]. We fix a faithful tracial state $\tau$ and then have $\tau\left(P_{\lambda}\right) \in$ $[0,|V(\bar{\Gamma})|] \cap S$ for any gap projection $P_{\lambda}$. We thus see that there are only finitely many possible gaps if all the $\theta_{i j}$ are rational.

3.11. Geometry of $\boldsymbol{B}$. In general, we are given a lattice $\Lambda$ and perhaps the graph $\Gamma_{\Lambda}$. We can then obtain a family of BH-algebras by choosing different cocycles $\alpha_{2 \pi \hat{\Theta}}$. We will call an element of this family $\mathscr{B}_{\widehat{\Theta}}$. Now we have already shown that such an algebra has a faithful matrix representation $\mathscr{B}_{\Theta} \subset M_{k}\left(\mathbb{T}_{\Theta}^{n}\right)$ where $k$ depends on $\Gamma$. It is interesting to note that this family of subalgebras has different geometries and $\mathrm{K}$-theories depending on the choice of $\Theta$. Generically one would expect

Expectation 3.20. If $\Theta$ is generic (i.e., all entries are irrational), then $\mathscr{B}_{\Theta}=$ $M_{|V(\bar{\Gamma})|}\left(\mathbb{T}_{\Theta}^{n}\right)$, which is Morita equivalent to $\mathbb{T}_{\Theta}^{n}$.

Whether this expectation is met is of course dependent on the choices. It is true for all the cases we will study. The main motivation is that the noncommutative torus at generic $\Theta$ is simple, i.e., there are no two-sided ideals. This usually allows one to find that all the elementary matrices are in the algebra and hence the algebra is the full matrix ring. The details of our particular calculations given in $\S 4$ also illuminate this expectation.

An open question is what happens at non-generic values of $\Theta$, i.e., if one or more of the entries of $\Theta$ are rational. This again heavily depends on the entries of $H_{\Lambda}$. In the cases we study below either $\mathscr{B}_{\Theta}=M_{\mid V(\bar{\Gamma} \mid}\left(\mathbb{T}_{\Theta}^{n}\right)$ again, or it is a genuine subalgebra. This is for instance a good new source of such algebras and for families in which the K-theory may jump.

The commutative case $\mathscr{B}_{0}$ is also very interesting. Here we can characterize the $\mathrm{C}^{*}$-algebra $\mathscr{B}_{0}$ by the space it represents via the Gelfand-Naimark theorem. For this we need some terminology. For each character or $\mathrm{C}^{*}$-algebra morphism $\chi: \mathbb{T}^{n} \rightarrow \mathbb{C}$, there is an induced $\mathrm{C}^{*}$-algebra morphism $\bar{\chi}: M_{k}\left(\mathbb{T}^{n}\right) \rightarrow M_{k}(\mathbb{C})$ for any $k$. We fix $k=|V(\bar{\Gamma})|$. We say $H_{\Lambda}$ is generic if it has $k$ distinct eigenvalues in $\mathbb{T}^{n}$ or, equivalently, if there is a character $\chi$ of $\mathbb{T}^{n}$ such that $\bar{\chi}(H)$ has $k$ distinct eigenvalues.

We call a point $\chi$ of $\mathbb{T}^{n}$ degenerate if $\bar{\chi}(H)$ has eigenvalues with higher multiplicities. The action of $L$ gives an inclusion $i: \mathbb{T}^{n} \rightarrow \mathscr{B}_{0}$. Given a character of $\mathscr{B}_{0}$, we also get a character of $\mathbb{T}^{n}$ by pull-back along $i$. We call a point $\chi$ of $\mathcal{B}_{0}$ degenerate if $\overline{i^{*}(\chi)}(H)$ has eigenvalues with higher multiplicity.

Theorem 3.21. If $H_{\Lambda}$ is generic, then $\mathscr{B}_{0}=C^{*}(X)$, where $X$ is a generically $k$-fold cover of the torus $T^{n}$. This cover is ramified over the locus of degenerate points and is moreover a quotient of the trivial $k$-fold cover. Here the identifications are along degenerate points of $X$. 
Proof. First observe that the trivial $k$-fold cover of $T^{n}$ has the $\mathrm{C}^{*}$-algebra $\mathbb{T}^{n}\left[e_{1}, \ldots, e_{k}\right] / R$, where the $e_{i}$ are self-adjoint and generate a semisimple algebra. This means that the relations $R$ are equivalent to the equations $e_{i} e_{j}=\delta_{i j} e_{i}$ and $\sum e_{i}=1$. Here the $e_{i}$ can be understood as the projectors to each of the copies.

Secondly we characterize $\mathcal{B}_{0}$. It is certainly a quotient of the $\mathrm{C}^{*}$-algebra $\mathbb{T}[H]$, where $H$ is a new self-adjoint generator which commutes with the previous generators. The way to understand its quotient is as follows. By the theorem of Caley-Hamilton we know that the characteristic polynomial $p$ of $H$ annihilates $H$ : $p(H)=0$. We claim this is the only relation. Indeed if there were any other relation $r$, then we could write $r=r^{\prime}+r^{\prime \prime}$ with $r^{\prime} \in(p(H))$ and $r^{\prime \prime}$ a polynomial in $H$ of degree less than $k$. This relation would hold after applying any character $\chi$, i.e., $\bar{\chi}(r)=0$. Since $\bar{\chi}\left(r^{\prime}\right)=0$ we also get $\bar{\chi}\left(r^{\prime \prime}\right)=0$. But generically there are $k$ distinct eigenvalues, so that for generic choices of $\chi$ the minimal polynomial of $\bar{\chi}(H)$ is the characteristic polynomial. Thus, as a polynomial in $H$, the degree of $r^{\prime \prime}$ must be bigger or equal to $k$, which means that $r^{\prime \prime}=0$. Hence $\mathscr{B}_{0}=\mathbb{T}^{n}[H] / p[H]$.

We can now give the $\mathrm{C}^{*}$-morphisms corresponding to the geometric continuous maps:

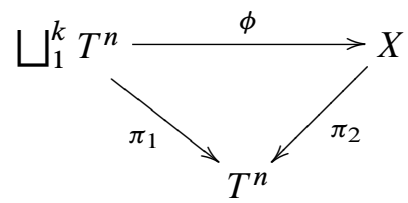

Let $\lambda_{i} \in \mathbb{T}^{n}$ be the eigenvalues of $H$ as a matrix with coefficients in the commutative ring $\mathbb{T}^{n}$. Then the map $\phi$ is given by $H \mapsto \sum \lambda_{i} e_{i}$. The maps $\pi_{1}$ and $\pi_{2}$ are just the inclusion maps. There are sections $s_{k}$ of $\pi_{1}$, given by sending $e_{j} \mapsto \delta_{j, k} 1$, which give rise to sections $\tilde{s}_{k}=\phi \circ s_{k}$. The corresponding $\mathrm{C}^{*}$-map is given by $H \mapsto \lambda_{k}$. From this the claims follow readily.

\section{Results for the Bravais, honeycomb and gyroid lattices}

\subsection{The Bravais lattice cases}

4.1.1. The $\mathbb{Z}^{n}$ case. In case $\Lambda=\mathbb{Z}^{n}$, we see that $L=T(\Lambda)=\mathbb{Z}^{n}$ and $\bar{\Gamma}_{\Lambda}$ is the graph with one vertex and $n$ loops.

From the graph $\bar{\Gamma}_{\Lambda}$ we can already read off that $\pi_{1}\left(\Gamma_{\Lambda}\right)=\left[\mathbb{F}_{n}, \mathbb{F}_{n}\right]$ according to Proposition 3.5, since the condition is obviously satisfied. Minimal loops are of length 4 and there are $\left(\begin{array}{l}n \\ 2\end{array}\right)$ unoriented loops.

Fixing a cocycle $\alpha$ by fixing an anti-symmetric matrix $\Theta$ (recall that this is equivalent to fixing a constant $B$-field), the corresponding Harper operator is just

$$
H_{\mathbb{Z}^{n}}=\sum_{i=1}^{n} U_{e_{i}}+U_{e_{i}}^{-1}=\sum_{i=1}^{n} U_{i}+U_{i}^{*},
$$


where $e_{i}$ are the standard unit basis vectors of $\mathbb{R}^{n}$ and $U_{i}$ are the generators of the noncommutative $n$-torus $\mathbb{\mathbb { T }}_{\Theta}^{n}$.

The algebra generated by the representation of $L=\mathbb{Z}^{n}$ is just the noncommutative torus $\mathbb{T}_{\Theta}^{n}$, and since $H \in \mathbb{T}_{\Theta}^{n}$, the algebra $\mathscr{B}_{\Theta}$ is also the noncommutative torus $B_{\Theta}=\mathbb{T}_{\Theta}^{n}$.

4.1.2. Other Bravais lattices. If $\Lambda$ is the set of points of a Bravais lattice, then again $L=T(\Lambda)=\Lambda$. For the graph $\bar{\Gamma}_{\Lambda}$ we need the information which of the distances between vertices of $\Lambda$ are minimal, or we need the additional data of a graph $\Gamma_{\Lambda}$. This information is also crucial in determining $\pi_{1}$.

Let $e_{j}, j \in J$, be the collection of these minimal vectors and fix an orientation $\vec{e}_{j}$ for each of them. As $\Lambda=L$ is a subgroup, $0 \in \Lambda$, and the minimal vectors are given by the $\lambda \in \Lambda$ with minimal length. Again fix a cocycle $\alpha$ by fixing the anti-symmetric matrix $\Theta$. Then

$$
H_{\Lambda}=\sum_{j \in J} U_{\vec{e}_{j}}+U_{\vec{e}_{j}}^{*} .
$$

The algebra generated by $L$ is always $\mathbb{T}_{\Theta}^{n}$, and since $H_{\Lambda} \in \mathbb{T}_{\Theta}^{n}$, we again obtain $\mathscr{B}_{\Theta}=\mathbb{T}_{\Theta}^{n}$.

Example 4.1. The triangular lattice. This is the lattice spanned by the vectors $e_{1}=$ $(1,0)$ and $e_{2}=\left(\frac{1}{2}, \frac{\sqrt{3}}{2}\right)$ in $\mathbb{R}^{2}$. In this case the graph $\bar{\Gamma}_{\Lambda}$ has one vertex and three loops with the six oriented edges corresponding to $\pm e_{1}, \pm e_{2}, \pm\left(e_{1}-e_{2}\right)$. Hence the condition of Proposition 3.5 is not met. One can compute the fundamental group by elementary methods.

The choice of $\Theta$ is given by $\Theta=\theta\left(\begin{array}{cc}0 & 1 \\ -1 & 0\end{array}\right)$. The Harper operator is given by

$$
\begin{aligned}
H_{\Lambda_{\mathrm{tri}}} & =U_{e_{1}}+U_{e_{1}}^{*}+U_{e_{2}}+U_{e_{2}}^{*}+U_{e_{1}-e_{2}}+U_{e_{1}-e_{2}}^{*} \\
& =U_{1}+U_{1}^{*}+U_{2}+U_{2}^{*}+U_{3}+U_{3}^{*},
\end{aligned}
$$

where $U_{1} U_{2} U_{3}=e^{i \pi \theta}$ id. Since $H \in \mathbb{T}_{\theta}^{2}$, the BH-algebra is $\mathscr{B}_{\Theta}=\mathbb{T}_{\theta}^{2}$ in the notation of Example 3.12.

\subsection{The honeycomb lattice}

4.2.1. Classical geometry. The honeycomb lattice $\Lambda=\Lambda_{\text {hon }}$ is the two-dimensional lattice specified in \$3.6.1. Its quotient graph $\bar{\Gamma}_{\Lambda}$ is the graph with two vertices and three edges depicted in Figure 8.

We set $e_{1}=(-1,0), e_{2}=\frac{1}{2}(1, \sqrt{3})$ and $e_{3}=\frac{1}{2}(1,-\sqrt{3})$. We choose the rooted spanning tree $\tau$ (root $A$, sole edge $e_{1}$ as indicated in Figure 8). The loops $\alpha$ and $\beta$ lift as $l_{2}=e_{2}\left(-e_{1}\right)$ and $l_{3}=e_{3}\left(-e_{1}\right)$. We see that $\vec{l}_{2}=\frac{1}{2}(-3, \sqrt{3})$ and $\vec{l}_{3}=\frac{1}{2}(3, \sqrt{3})$. Thus the condition of Proposition 3.5 is met and we obtain 


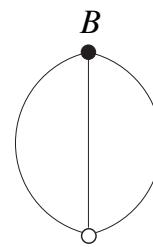

A

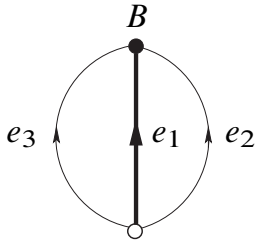

A

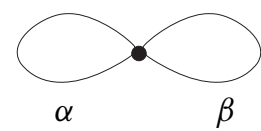

Figure 8 . The graph $\bar{\Gamma}_{\Lambda}$ for the honeycomb lattice, the spanning tree $\tau$ (root $A$, sole edge $e_{1}$ ) together with a set of oriented edges, the quotient graph $\bar{\Gamma}_{\Lambda} / \tau$.

Proposition 4.2. We have

$$
\pi_{1}\left(\Gamma_{\Lambda}\right)=\left[\mathbb{F}_{2}, \mathbb{F}_{2}\right] .
$$

At each point of the lattice there are six directed or three undirected minimal loops of length 6.

Proof. It is clear by inspection that there are no loops of length 2 and 4, and the loops through an oriented edge twice will give length bigger than 6 . To get a minimal loop, we need to pass through each edge exactly once in each direction, if possible. This can be done by fixing the first edge, and then choosing among the two left over edges the second returning edge. Now everything is fixed. One has to leave the vertex by the last edge and one concludes a cycle of six edges by choosing the only possible non-traversed edge oriented without using an oriented edge twice. This of course gives the known three unoriented loops at each vertex.

Remark 4.3. In the generators $\alpha, \beta$, these loops are given by the elements $\left[l_{2}, l_{3}\right]^{ \pm 1}$, $\left[l_{2}^{-1}, l_{3}\right]^{ \pm 1}$ and $\left[l_{2}, l_{3}^{-1}\right]^{ \pm 1}$.

It is interesting to note that the map $\bar{\Gamma}_{\Lambda} \rightarrow \bar{\Gamma}_{\Lambda} / \tau$ induces a new length function on the free group $\mathbb{F}_{2}$ which is not symmetric in symmetric generators. For instance, the commutator $\left[l_{2}^{-1}, l_{3}^{-1}\right]$ has length 8 in this metric, whereas all other commutators listed above have length 6 in the generators $e_{i}$.

Remark 4.4. There is another observation. Given a loop on $\Gamma_{\Lambda}$ it decomposes into blocks of loops on $\bar{\Gamma}_{\Lambda}$. Here we could take the loop $l_{1}=e_{1}\left(-e_{2}\right) e_{3}\left(-e_{1}\right) e_{2}\left(-e_{3}\right)$ which decomposes into 3 blocks. Now cyclically permuting these blocks, we also get a loop on $\Gamma_{\Lambda}$, and of course the inverse of any loop is a loop so that the loop $l_{1}$ generates all six loops.

4.2.2. Quantum geometry. Fixing a constant magnetic field $B=2 \pi \widehat{\Theta}$ amounts to choosing an anti-symmetric bilinear form $\widehat{\Theta}$ on $\mathbb{R}^{2}$. We will use the induced cocycle both on $L$ and on $T(\Lambda)$. The matrix $\Theta$ is the matrix of $\widehat{\Theta}$ with respect to the basis $\left(f_{2}, f_{3}\right)$ of $L$. It is completely determined by its value $\widehat{\Theta}\left(f_{2}, f_{3}\right)$. The cocycle induced on $T(\Lambda)$ will also play a role. It is fixed by the value $\Theta\left(e_{1}, e_{2}\right)$. We fix the 
notation that

$$
\theta:=\widehat{\Theta}\left(f_{2}, f_{3}\right), \quad q:=e^{2 \pi i \theta} \quad \text { and } \quad \phi=\widehat{\Theta}\left(-e_{1}, e_{2}\right), \quad \chi:=e^{i \pi \phi} .
$$

The values of $\chi$ and $\theta$ are not independent:

$$
\theta=-3 \phi \text { and } q=\bar{\chi}^{6}
$$

This follows from the elementary calculation

$$
\begin{aligned}
\theta=\widehat{\Theta}\left(f_{2}, f_{3}\right) & =\widehat{\Theta}\left(e_{2}-e_{1}, e_{3}-e_{1}\right) \\
& =\widehat{\Theta}\left(e_{2}, e_{3}\right)+\widehat{\Theta}\left(e_{2},-e_{1}\right)+\widehat{\Theta}\left(-e_{1}, e_{3}\right)+\widehat{\Theta}\left(-e_{1},-e_{1}\right) \\
& =\widehat{\Theta}\left(e_{2},-e_{1}\right)-\phi+\widehat{\Theta}\left(-e_{1},-e_{2}\right)=-3 \phi
\end{aligned}
$$

The Hilbert space $\mathscr{H}=l^{2}\left(\Lambda_{\text {hon }}\right)$ splits

$$
\mathscr{H}_{\text {hon }}=\mathscr{H}_{A} \oplus \mathscr{H}_{B},
$$

where $A$ and $B$ are the vertices as indicated in Figure 8. The six oriented edges $\pm e_{i}$, $i=1, \ldots 3$, of $\bar{\Gamma}_{\Lambda}$ give rise to three partial isometry operators $U_{ \pm i}$ :

$$
U_{i}:=\left(\begin{array}{cc}
0 & 0 \\
U_{e_{i}} & 0
\end{array}\right), \quad U_{-i}:=\left(\begin{array}{cc}
0 & U_{-e_{i}} \\
0 & 0
\end{array}\right),
$$

where $U_{e_{i}}$ and $U_{-e_{i}}=U_{e_{i}}^{-1}=U_{e_{i}}^{*}$ are the isomorphisms as in $\S 3.8$.

The Harper Hamiltonian now reads:

$$
H_{\Lambda}=\sum_{i=1}^{3} U_{i}+U_{i}^{-1}=\left(\begin{array}{cc}
0 & U_{e_{1}}^{*}+U_{e_{2}}^{*}+U_{e_{3}}^{*} \\
U_{e_{1}}+U_{e_{2}}+U_{e_{3}} & 0
\end{array}\right)
$$

In order to put it into a matrix form with coefficients in $T_{\theta}^{2}$, we again choose the spanning tree $\tau$ as indicated in Figure 8 .

The Hamiltonian now reads

$$
H_{\Lambda, \tau}=\left(\begin{array}{cc}
0 & 1+U^{*}+V^{*} \\
1+U+V & 0
\end{array}\right) \in M_{2}\left(\mathbb{\mathbb { T }}_{\theta}^{2}\right),
$$

where we have used the operators $U:=\chi U_{f_{2}}$ and $V=\bar{\chi} U_{f_{3}}$. We have

$$
U V=q V U \text { or } \quad U V U^{*}=q V .
$$

The symmetry algebra generated by the translations $U_{f_{i}}$ is isomorphic to $\mathbb{T}_{\theta}^{2}$ on $\mathscr{H}_{A} \oplus \mathscr{H}_{A}$. It acts via the representation defined by $\rho\left(U_{f_{2}}\right)=\operatorname{diag}\left(U_{f_{2}}, \chi^{2} U_{f_{2}}\right)=$ $\operatorname{diag}(\bar{\chi} U, \chi U)$ and $\rho\left(U_{f_{3}}\right)=\operatorname{diag}\left(U_{f_{3}}, \bar{\chi}^{2} U_{f_{3}}\right)=\operatorname{diag}(\chi V, \bar{\chi} V)$.

Proposition 4.5. If $q \neq \pm 1$, then $\mathcal{B}_{\Theta}=M_{2}\left(\mathbb{\nabla}_{\theta}^{2}\right)$ and hence is Morita equivalent to $\mathbb{T}_{\theta}^{2}$. 
Proof. The strategy of proof is to show that the elementary matrix $E_{12} \in \mathcal{B}_{\Theta}$. If this happens, we get that all elementary matrices are in $\mathscr{B}_{\Theta}$, since $E_{21}=E_{12}^{*}$, $E_{11}=E_{12} E_{21}$ and $E_{22}=E_{21} E_{12}$ and then $\mathscr{B}_{\Theta}=M_{2}\left(\mathbb{T}_{\theta}^{2}\right)$.

The method to obtain $E_{12}$ is by direct calculation using the commutation relations (10).

The first step is to set $X=\rho\left(\bar{\chi}^{2} U\right) \rho\left(\bar{\chi}^{2} V^{*}\right) H \rho(V) \rho\left(U^{*}\right)$ and then set $X_{1}=$ $\frac{1}{1-\bar{q}}[H-\bar{q} X]$. Note we are using the assumption $q \neq 1$. Then

$$
X_{1}=\left(\begin{array}{cc}
0 & \frac{1}{1-\bar{q}}\left[\left(1-\bar{\chi}^{2}\right)+\left(1-\bar{\chi}^{4}\right) U^{*}+\left(1-\chi^{4}\right) V^{*}\right. \\
1 & 0
\end{array}\right) .
$$

In step two, we set $X_{2}=H-X_{1} \rho(1+U+V)=\left(\begin{array}{cc}0 & * * \\ 0 & 0\end{array}\right)$, where explicitly

$$
* *=B U^{*}+C V^{*}+D U+E V+F U^{*} V+G V^{*} U
$$

with

$$
\begin{gathered}
B=C=\frac{\chi^{4}\left(1-\chi^{2}\right)}{1-\chi^{6}}, \quad D=\frac{1-\chi^{2}}{1-\chi^{6}}, \quad E=\frac{\bar{\chi}^{2}\left(\bar{\chi}^{2}-1\right)}{1-\chi^{6}}, \\
F=\frac{\chi^{2}\left(1-\bar{\chi}^{4}\right)}{1-\chi^{6}}, \quad G=\frac{\chi^{2}\left(\chi^{4}-1\right)}{1-\chi^{6}} .
\end{gathered}
$$

Notice that $\chi^{6}=\bar{q} \neq 1$ by assumption. The coefficients do not vanish if $\chi^{4} \neq 1$ and $\chi^{2} \neq 1$. But $\chi^{2}=1$ implies that $\chi^{6}=1$, and $\chi^{4}=1$ implies that $\chi^{12}=1$ and so $\chi^{6}=q= \pm 1$. If $q \neq \pm 1$, then all the coefficients are non-zero. We can obtain $E_{12}$ in several steps by setting $X_{3}=X_{2}-\rho(U) X_{2} \rho\left(U^{*}\right), X_{4}=X_{3}-\rho(V) X_{3} \rho\left(V^{*}\right)$, $X_{5}=\rho(U) X_{4} \rho\left(U^{*}\right)-q X_{4}$ and finally obtain $E_{12}=X_{5}-\rho\left(\frac{1}{(\bar{q}-q)\left(1-\bar{q}^{2}\right)} U^{*} V\right) X_{5}$, where for the last step we need the assumption $q \neq-1$.

The situation for $q=-1$ is more complicated. Notice that in this case $\mathbb{T}_{\frac{1}{2}}^{2}$ is not simple. For instance, there is a $*$ homomorphism of $\phi: \mathbb{T}_{\frac{1}{2}}^{2} \rightarrow \operatorname{Cliff}\left(-\mathrm{Id}_{2}\right) \otimes \mathbb{C}$, where Cliff $\left(-\mathrm{Id}_{2}\right) \otimes \mathbb{C}$ is the Clifford algebra over $\mathbb{C}$ of the standard quadratic form given by the negative of the $(2 \times 2)$-identity matrix $\operatorname{Id}_{2}$. If $i$ and $j$ are the usual basis vectors, then $\phi(U)=i, \phi(V)=j$.

We let $g:=\operatorname{ker} \phi=\left\langle 1+U^{2}, 1+V^{2}\right\rangle$.

There is also an algebra involution $\wedge$ on $\mathbb{T}_{\frac{1}{2}}^{2}$ given by $\widehat{U}=-V, \widehat{V}=-V$.

Proposition 4.6. If $q=-1$ and $\chi^{4} \neq 1$, then $\mathscr{B}_{\Theta}=M_{2}\left(\mathbb{T}_{\frac{1}{2}}^{2}\right)$. If $q=-1$ and $\chi^{4}=1$, then $\mathscr{B}_{\Theta}$ is the subalgebra of $M_{2}\left(\mathbb{T}_{\frac{1}{2}}^{2}\right)$ given by matrices of the form

$$
\left(\begin{array}{ll}
a & b \\
\hat{b} & \hat{a}
\end{array}\right)+J, \quad a, b \in \mathbb{T}_{\frac{1}{2}}^{2}, J \in M_{2}(\mathcal{g}) .
$$


Proof. In the case $q=-1$ we can at first proceed as in the proof of Proposition 4.5. As $X_{1}$ we obtain

$$
X_{1}=\left(\begin{array}{cc}
0 & \frac{1}{2}\left[\left(1-\bar{\chi}^{2}\right)+\left(1-\chi^{4}\right)\left(U^{*}+V^{*}\right)\right] \\
1 & 0
\end{array}\right)
$$

Here the two cases split dependent on whether $\chi^{4}=1$ or $\chi^{4} \neq 1$.

We will deal with the case $\chi^{4} \neq 1$ first. We set $\tilde{X}_{2}=\widetilde{X}_{1}-\rho(U) \tilde{X}_{1} \rho\left(\chi^{2} U^{*}\right)$, $\tilde{X}_{3}=\tilde{X}_{2}+\rho(U) \tilde{X}_{2} \rho\left(\chi^{2} U^{*}\right)$. Then $\frac{1}{\left(1-\chi^{4}\right)\left(1+\chi^{4}\right)} \tilde{X}_{3}=E_{21}$, hence we get $E_{12}=$ $E_{12}^{*} \in \mathscr{B}_{\Theta}$, and so $\mathscr{B}_{\Theta}$ is the full matrix algebra.

If $q=-1$ and $\chi^{4}=1$, then, since $q=\bar{\chi}^{6}=-1$, we get $\bar{\chi}^{2}=-1$ and $X_{1}=I=E_{12}+E_{21} \in \mathcal{B}_{\Theta}$. Furthermore, we get

$$
X_{2}=\left(\begin{array}{cc}
0 & \left.1+U^{*}+V^{*}-U-V\right] \\
1 & 0
\end{array}\right) .
$$

Setting $Y_{3}:=\frac{1}{2} \rho(U)\left[X_{2}+\rho(U) X_{2} \rho\left(-U^{*}\right)\right]$ and $\tilde{Y}_{3}=\frac{1}{2} \rho(V)\left[X_{2}-\rho(U) X_{2} \rho\left(-U^{*}\right)\right.$ we get

$$
Y_{3}=\left(\begin{array}{cc}
0 & 1+U^{2} \\
0 & 0
\end{array}\right) \in \mathcal{B}_{\Theta}, \quad \tilde{Y}_{3}=\left(\begin{array}{cc}
0 & 1+V^{2} \\
0 & 0
\end{array}\right) \in \mathcal{B}_{\Theta} .
$$

Let $\mathscr{B}^{\prime}$ be the algebra above given in (11). It is easy to check that $\mathscr{B}^{\prime}$ is indeed a C*-algebra since $M_{2}(\mathcal{A})$ is a two-sided ideal of $M_{2}\left(T_{\frac{1}{2}}^{2}\right)$ and $\wedge$ commutes with $*$. Now $U^{*}+U$ and $V^{*}+V$ are both in $\mathcal{g}$ so that

$$
H=\left(\begin{array}{cc}
0 & 1+U^{*}+V^{*} \\
1-U^{*}-V^{*} & 0
\end{array}\right)+\left(\begin{array}{cc}
0 & 0 \\
U+U^{*}+V+V^{*} & 0
\end{array}\right) \in \mathscr{B}^{\prime} .
$$

Furthermore certainly the operators of $L$ are in $\mathcal{B}^{\prime}$ so that the inclusion $\mathcal{B}_{\Theta} \subset \mathscr{B}^{\prime}$ holds.

On the other hand, since $L \subset \mathscr{B}_{\Theta}$, all the matrices $\operatorname{diag}(a, \hat{a})$ are in $\mathcal{B}_{\Theta}$ and by the above $I \in \mathscr{B}_{\Theta}$ so that all matrices of the form $\left(\begin{array}{ll}a & b \\ \hat{b} & \hat{a}\end{array}\right)$ are in $\mathscr{B}_{\Theta}$. Furthermore since the matrices $\left(1+U^{2}\right) E_{21}$ and analogously $\left(1+V^{2}\right) E_{21}$ are in $\mathscr{B}_{\Theta}$, taking products with $I=E_{12}+E_{21}$, we obtain that all the matrices of $M_{2}(\mathcal{A}) \subset \mathcal{B}_{\Theta}$. Hence $\mathcal{B}^{\prime} \subset \mathcal{B}_{\Theta}$, as claimed.

We now analyze the case of $q=1$. Let $(x)$ be the principal (algebra) ideal generated by $x$ in $\mathbb{T}_{0}^{2}$. Set $J_{1}=(1+U+V), J_{2}=\left(1+U^{*}+V^{*}\right)$ and $J_{12}=J_{1} J_{2}$.

Proposition 4.7. If $q=1$ and $\chi= \pm 1$, then $\mathcal{B}_{\Theta}=C^{*}(X)$, where $X$ is the double cover of the torus $S^{1} \times S^{1}$ ramified at the points $\left(e^{2 \pi i \frac{1}{3}}, e^{2 \pi i \frac{2}{3}}\right)$ and $\left(e^{2 \pi i \frac{1}{3}}, e^{2 \pi i \frac{2}{3}}\right)$.

If $q=1$ and $\chi \neq \pm 1$, then $\mathcal{B}_{\Theta}$ is equal to the matrix $C^{*}$-subalgebra of $M_{2}\left(\mathbb{T}_{0}^{2}\right)$ given by matrices in the $C^{*}$-subalgebra

$$
\rho\left(\mathbb{\nabla}^{2}\right)+\left(\begin{array}{cc}
J_{12} & J_{2} \\
J_{1} & J_{12}
\end{array}\right)
$$


Proof. The first statement follows from Theorem 3.21 for $\chi=1$. The eigenvalues of $H$ in $\mathbb{T}_{0}^{2}$ are $\pm \sqrt{(1+U+V)\left(1+U^{*}+V^{*}\right)}$, which exist by continuous operator calculus since the operator under the square root is self-adjoint of the form $A A^{*}$ and hence has nonnegative real spectrum. The points of the space $X$ equivalent to $\mathscr{B}_{0}$ where these two eigenvalues coincide are given by precisely the points above.

For $\chi=-1$, we remark that $\mathcal{B}_{\Theta}=\mathscr{B}_{0}$, by using the involution $U \mapsto-U$, $V \mapsto-V$.

If $q=1$, but $\chi \neq \pm 1$, we actually know that $\chi$ is a sixth root of unity and $\chi^{2}=\zeta_{3}=e^{2 \pi i \frac{1}{3}}$ or $\chi^{2}=\zeta_{3}^{2}$, and in these cases $\chi^{4}=\chi \neq 1$. Let $\mathscr{B}^{\prime}$ be the algebra of (12). Since $J_{1}^{*}=J_{2}$ and both of them are algebra ideals, it is clear that $\mathscr{B}^{\prime}$ is a $\mathrm{C}^{*}$-subalgebra. Then it is clear that $\mathscr{B}_{\Theta} \subset \mathscr{B}^{\prime}$. In order to prove the reverse inclusion, we notice that $\frac{1}{1-\chi}\left[H-\rho(U) H \chi^{2} \rho\left(U^{*}\right)\right]=(1+U+V) E_{21} \in \mathcal{B}_{\Theta}$. Hence also $\left(1+U^{*}+V^{*}\right) E_{21} \in \mathscr{B}_{\Theta}$ as well as $(1+U+V)\left(1+U^{*}+V^{*}\right) E_{11} \in \mathscr{B}_{\Theta}$ and $(1+U+V)\left(1+U^{*}+V^{*}\right) E_{22} \in \mathscr{B}_{\Theta}$. This together with the action of $L$ shows that $\mathscr{B}^{\prime} \subset \mathscr{B}_{\Theta}$.

In the notation of equation (9):

Theorem 4.8. If $q \neq \pm 1$, the algebra $\mathscr{B}_{\Theta}$ has the $K_{0}$-group $\mathbb{Z} \oplus \mathbb{Z}$. If $\chi= \pm 1$, then $K_{0}\left(\mathcal{B}_{\Theta}\right)=\mathbb{Z}^{3}$.

Proof. For $\alpha \neq 1$ this directly follows from Proposition 4.5 below. For the commutative case $q=1, \chi= \pm 1$, this follows from the fact that the double cover of the torus obtained by identifying two pairs of points of two tori has the corresponding $K_{0}$-group.

\subsection{The gyroid}

4.3.1. The lattice and sublattice Hilbert spaces. The lattice $\Gamma_{+}$has the Hilbert space $\mathscr{H}_{\Gamma_{+}}=l^{2}\left(\Gamma_{+}\right)$.

The subspace $\mathscr{H}_{\Gamma_{+}}$decomposes naturally into subspaces $\mathscr{H}_{i}$ where $\mathscr{H}_{i}=\mathscr{H}_{\mathbb{Z}^{3}\left(v_{i}\right)}$ and $\mathbb{Z}^{3}\left(v_{i}\right)$ denotes the set of all translates under $\mathbb{Z}^{3}$ of $v_{i}$. Thus $\mathscr{H}_{\Gamma}=\bigoplus_{i=0}^{7} \mathscr{H}_{i}$. This is the composition corresponding to $\bar{\Gamma}_{+}^{\text {crystal }}$. In order to write down the Hamiltonian effectively, we will put together the summands in pairs:

$$
\mathscr{H}_{\Gamma}=\mathscr{H}_{A} \oplus \mathscr{H}_{B} \oplus \mathscr{H}_{C} \oplus \mathscr{H}_{D},
$$

where $\mathscr{H}_{A}=\mathscr{H}_{0} \oplus \mathscr{H}_{6}, \mathscr{H}_{B}=\mathscr{H}_{1} \oplus \mathscr{H}_{7}, \mathscr{H}_{C}=\mathscr{H}_{3} \oplus \mathscr{H}_{5}, \mathscr{H}_{D}=\mathscr{H}_{2} \oplus \mathscr{H}_{4}$.

This corresponds to passing from $\bar{\Gamma}_{+}^{\text {crystal }}$ to $\bar{\Gamma}_{+}$and to our general setup of graph Hamiltonians. 
4.3.2. Cocycles for $\mathbb{R}^{\mathbf{3}}$. As discussed, choosing a magnetic field corresponds to a projective representation of $\Gamma_{+}$by unitary operators on $\mathcal{H}_{\Gamma_{+}}$with a cocycle $\alpha$. We recall this here in a more familiar three-dimensional setting.

A skew-symmetric bilinear form $B$ translates to a more well-known expression as follows. Let $i, j, k$ be the standard unit vectors on $\mathbb{R}^{3}$, and set $B_{x}=B(j, k)$, $B_{y}=B(k, i)$ and $B_{z}=B(i, j)$. If $\vec{B}=\left(B_{x}, B_{y}, B_{z}\right)$, then

$$
B\left(m, m^{\prime}\right)=\vec{B} \cdot\left(m \times m^{\prime}\right), \quad \alpha_{B}\left(m, m^{\prime}\right)=\exp \left(i \frac{1}{2} \vec{B} \cdot\left(m \times m^{\prime}\right)\right) .
$$

Indeed $\alpha_{B}$ is a cocycle since

$$
\begin{aligned}
B \cdot\left(m \times m^{\prime}\right)+B \cdot\left(\left(m+m^{\prime}\right) \times m^{\prime \prime}\right) & =B \cdot\left(m \times m^{\prime}+m \times m^{\prime \prime}+m^{\prime} \times m^{\prime \prime}\right) \\
& =B \cdot\left(m \times\left(m^{\prime}+m^{\prime \prime}\right)\right)+B \cdot\left(m^{\prime} \times m^{\prime \prime}\right) .
\end{aligned}
$$

The physics interpretation of this is that $B$ is a fixed constant magnetic field, and then $\alpha_{B}\left(m, m^{\prime}\right)$ is the magnetic flux through the triangle spanned by $m$ an $m^{\prime}$.

4.3.3. Cocycles for the gyroid. The maximal translation group $L$ for $\Gamma_{+}$is the bcc lattice spanned by the vectors $f_{i}$ given in (2) or $g_{i}$ (3). The lattice group $T\left(\Gamma_{+}\right)$ is the fcc lattice spanned by the vectors $e_{4}, e_{5}, e_{6}$. By restricting a cocycle given by a vector $\vec{B}$ on $\mathbb{R}^{3}$, we obtain a cocycle for each of these lattices. Since both the fcc and the bcc lattices are Bravais lattices, these are precisely all cocycles coming from cocycles based on anti-symmetric forms $\widehat{\Theta}=\frac{1}{2 \pi} B$ stemming from a magnetic field $B$.

We will use the basis $\left(g_{i}\right)$ to fix the matrix representation $\Theta$ of $\widehat{\Theta}$.

4.3.4. Graph representation and graph Harper operator on $\mathscr{H}_{\Gamma_{+}}$. We fix a vector $B$. This fixes the corresponding cocycle $\alpha(v, w):=e^{i \frac{1}{2} B \cdot(v \times w)}$. Now consider the graph representation as defined in $\$ 3.10$, where the order of the vertices is $A, B$, $C, D$.

Using the fixed cocycle $\alpha$ above, we obtain the partial isometries corresponding to elements of $T\left(\Gamma_{+}\right)$. These are $U_{i}:=U_{e_{i}}$ according to the list (4) as discussed in $\$ 3.8$.

In Hilbert space decomposition (13) the graph Harper operator $H_{\bar{\Gamma}}$ becomes the $(4 \times 4)$-matrix

$$
H_{\bar{\Gamma}_{+}}=\left(\begin{array}{cccc}
0 & U_{1}^{*} & U_{2}^{*} & U_{3}^{*} \\
U_{1} & 0 & U_{6}^{*} & U_{5} \\
U_{2} & U_{6} & 0 & U_{4} \\
U_{3} & U_{5}^{*} & U_{4}^{*} & 0
\end{array}\right) .
$$

Recall that $U_{i}=\rho\left(e_{i}\right)$ and $U_{i}^{*}=U_{i}^{-1}=U_{-e_{i}}$. 
4.3.5. Matrix Harper operator. We choose the rooted spanning tree $\tau$ (root $A$, edges $\left.e_{1}, e_{2}, e_{3}\right)$ as indicated in Figure 5. Using this we obtain the matrix Harper operator

$$
H_{\bar{\Gamma}_{+}, \tau}=\left(\begin{array}{cccc}
0 & 1 & 1 & 1 \\
1 & 0 & U_{1}^{*} U_{6}^{*} U_{2} & U_{1}^{*} U_{5} U_{3} \\
1 & U_{2}^{*} U_{6} U_{1} & 0 & U_{2}^{*} U_{4} U_{3} \\
1 & U_{3}^{*} U_{5}^{*} U_{1} & U_{3}^{*} U_{4}^{*} U_{2} & 0
\end{array}\right)=:\left(\begin{array}{cccc}
0 & 1 & 1 & 1 \\
1 & 0 & A & B^{*} \\
1 & A^{*} & 0 & C \\
1 & B & C^{*} & 0
\end{array}\right)
$$

according to $\$ 3.10 .2$. The coefficients can be expressed in terms of the operators of the magnetic translation operators of the bcc lattice. We fix $U:=U_{f_{1}}, V:=U_{f_{2}}$ and $W:=U_{f_{3}}$ for the $f_{i}$ listed in (2). Then

$$
A=a V^{*} W, \quad B=b W U^{*}, \quad C=c W^{*} U V,
$$

where

$$
\begin{aligned}
a & =\frac{\alpha\left(e_{2},-e_{6}\right) \alpha\left(e_{2}-e_{6},-e_{1}\right)}{\alpha\left(-f_{2}, f_{3}\right)}, \\
b & =\frac{\alpha\left(-e_{3},-e_{5}\right) \alpha\left(-e_{3}-e_{5}, e_{1}\right)}{\alpha\left(f_{3},-f_{1}\right)}, \\
c & =\frac{\alpha\left(-e_{2}, e_{4}\right) \alpha\left(-e_{2}+e_{4}, e_{3}\right)}{\alpha\left(-f_{3}, f_{1}\right) \alpha\left(-f_{3}+f_{1}, f_{2}\right)} .
\end{aligned}
$$

4.3.6. Choices and notation. In order to proceed we fix some convenient basis and notation. First we change from the basis $f_{i}$ to the basis

$$
g_{1}=-f_{2}+f_{3}, \quad g_{2}=-f_{1}+f_{3}, \quad g_{3}=f_{1}+f_{2}-f_{3}
$$

for the bcc lattice $B$. We see that $A=a^{\prime} U_{g_{1}}, B=b^{\prime} U_{g_{2}}, C=c^{\prime} U_{g_{3}}$ again for fixed constants $a^{\prime}=\alpha\left(-e_{1},-e_{6}\right) \alpha\left(-e_{1}-e_{6}, e_{2}\right), b^{\prime}=\alpha\left(-e_{3},-e_{5}\right) \alpha\left(-e_{3}-e_{5}, e_{2}\right)$ and $c^{\prime}=-\alpha\left(-e_{2}, e_{4}\right) \alpha\left(-e_{2}+e_{4}, e_{3}\right)$. The operators $A, B, C$ again generate the $\mathrm{C}^{*}$-algebra of the noncommutative torus $\mathbb{T}_{\Theta}^{3}$, where $\Theta$ is the matrix of the bilinear form in the basis $g_{i}$. Explicitly:

$$
\theta_{12}=\frac{1}{2 \pi} B \cdot\left(g_{1} \times g_{2}\right), \quad \theta_{13}=\frac{1}{2 \pi} B \cdot\left(g_{1} \times g_{3}\right), \quad \theta_{23}=\frac{1}{2 \pi} B \cdot\left(g_{2} \times g_{3}\right) .
$$

We write $\alpha_{1}:=e^{2 \pi i \theta_{12}}=\alpha^{2}\left(g_{1}, g_{2}\right), \bar{\alpha}_{2}:=e^{2 \pi i \theta_{13}}=\alpha^{2}\left(g_{1}, g_{3}\right)$ and $\alpha_{3}:=$ $e^{2 \pi i \theta_{23}}=\alpha^{2}\left(g_{2}, g_{3}\right)$. This means for the commutators

$$
A B=\alpha_{1} B A, \quad A C=\bar{\alpha}_{2} C A, \quad B C=\alpha_{3} C B .
$$

4.3.7. Matrix action of $\boldsymbol{T}\left(\boldsymbol{\Gamma}_{+}\right)$. Given the choice of the spanning tree $\tau$ also determines the action of $T\left(\Gamma_{+}\right)$.

It will be convenient to introduce forth roots of the $\alpha_{i}$ :

$$
\phi_{1}=e^{\frac{\pi}{2} i \theta_{12}}, \quad \phi_{2}=e^{\frac{\pi}{2} i \theta_{31}}, \quad \phi_{3}=e^{\frac{\pi}{2} i \theta_{23}}, \quad \Phi=\phi_{1} \phi_{2} \phi_{3}
$$


and the matrices

$$
\begin{gathered}
\Lambda_{1}=\operatorname{diag}\left(1, \phi_{1}, \phi_{2}, \overline{\phi_{1} \phi_{2}}\right), \quad \Lambda_{2}=\operatorname{diag}\left(1, \phi_{1}, \overline{\phi_{1} \phi_{3}}, \phi_{3}\right), \\
\Lambda_{3}=\operatorname{diag}\left(1, \overline{\phi_{2} \phi_{3}}, \phi_{2}, \phi_{3}\right) .
\end{gathered}
$$

By definition $\alpha_{1} \alpha_{2} \alpha_{3}=\Phi^{4}$.

Then the action $\rho$ is given by the matrices

$$
\begin{aligned}
& \rho(A)=\Lambda_{1} \operatorname{diag}(A, A, A, A), \\
& \rho(B)=\Lambda_{2} \operatorname{diag}(B, B, B, B), \\
& \rho(C)=\Lambda_{3} \operatorname{diag}(C, C, C, C) .
\end{aligned}
$$

Note the following which makes the calculations quite a bit simpler:

$\Lambda_{1} \Lambda_{2}^{*}=\operatorname{diag}(1,1, \bar{\Phi}, \Phi), \quad \Lambda_{2} \Lambda_{3}^{*}=\operatorname{diag}(1, \Phi, \bar{\Phi}, 1), \quad \Lambda_{1} \Lambda_{3}^{*}=\operatorname{diag}(1, \Phi, 1, \bar{\Phi})$.

\subsubsection{Calculation of $\mathscr{B}_{\Theta}$ and $K_{*}\left(\mathscr{B}_{\Theta}\right)$}

Proposition 4.9. If $\Phi \neq 1$, then the $B H$-algebra $\mathcal{B}_{\Theta}$ is the full matrix algebra $\mathcal{B}_{\Theta}=M_{4}\left(\mathbb{T}_{\Theta}^{3}\right)$.

Proof. The strategy is to again show that enough elementary matrices are in $\mathcal{B}_{\Theta}$. This will be done in a case by case study. We will present the first case in detail.

Assume that $\alpha_{1} \neq \Phi^{2}, \alpha_{2} \neq \Phi$ and $\alpha_{3} \neq \Phi$. Then we have to do six steps to obtain $E_{34}$. These are

$$
\begin{aligned}
& X_{1}=H-\rho(A B *) H \rho\left(B A^{*}\right), \\
& X_{2}=X_{1}-\rho\left(B C^{*}\right) X_{1} \rho\left(C B^{*}\right), \\
& X_{3}=X_{2}-\rho\left(A C^{*}\right) X_{2} \rho\left(C A^{*}\right), \\
& X_{4}=\Phi \bar{\alpha}_{1} X_{3}-\rho\left(A B^{*}\right) X_{3} \rho\left(B A^{*}\right), \\
& X_{5}=\Phi \alpha_{3} X_{4}-\rho\left(B C^{*}\right) X_{4} \rho\left(C B^{*}\right), \\
& X_{6}=\Phi \bar{\alpha}_{2} X_{5}-\rho\left(A C^{*}\right) X_{5} \rho\left(C A^{*}\right) .
\end{aligned}
$$

The resulting matrix is

$$
\left(1-\bar{\Phi}^{2} \alpha_{2} \alpha_{3}\right)\left(1-\Phi \bar{\alpha}_{3}\right)\left(1-\bar{\Phi} \alpha_{2}\right)\left(\Phi \bar{\alpha}_{1}-\bar{\Phi}^{2} \alpha_{2} \alpha_{3}\right)\left(\bar{\Phi} \alpha_{3}-\Phi \bar{\alpha}_{3}\right)\left(\Phi \bar{\alpha}_{2}-\bar{\Phi} \alpha_{2}\right) E_{43},
$$

and the factor is invertible by assumption. This provides $E_{43}, E_{34}=E_{43}^{*}, E_{33}=$ $E_{34} E_{43}$ and $E_{44}=E_{43} E_{34}$. Now to get the other elementary matrices, we first assume that $\phi_{1} \neq 1$. Then set $Y_{1}=H E_{44}-\bar{\phi}_{3} \rho(C) E_{34}, Y_{2}=\phi_{3} Y_{1}-\rho\left(B^{*}\right) Y_{1} \rho(B)=$ $\phi_{3}\left(\bar{\phi}_{1}-1\right) E_{24}$ and $E_{14}=Y_{1}-\bar{\phi}_{3} \rho(B) E_{24}$, which guarantees that all the $E_{i j} \in \mathcal{B}_{\Theta}$. If $\phi_{1}=1$, then we use $Z_{2}^{\prime}=\bar{\phi}_{3} Y_{1}-\rho(C) H \rho\left(C^{*}\right)=\bar{\phi}_{3}\left(1-\alpha_{3} \bar{\Phi}\right) E_{24}$, so since $\alpha_{3} \neq \Phi$, we obtain $E_{24}$ and $E_{14}$ (as above) and hence again the whole matrix algebra. 
The arguments for the cases $\alpha_{1} \neq \Phi, \alpha_{2} \neq \Phi^{2}, \alpha_{3} \neq \Phi$ and $\alpha_{1} \neq \Phi, \alpha_{2} \neq \Phi$, $\alpha_{3} \neq \Phi^{2}$ are analogous.

Now by pure logic it follows that $\mathscr{B}_{\Theta}$ is the full matrix in all cases except the three cases $\alpha_{i}=\Phi^{2}, \alpha_{j}=\alpha_{k}=\Phi$ for $\{i, j, k\}=\{1,2,3\}$. We treat only one of these since the other two follow symmetrically. So assume that $\alpha_{1}=\Phi^{2}, \alpha_{2}=\alpha_{3}=\Phi$. In this case the story is similar but faster.

We keep $X_{1}, X_{2}$ as above. Then if $\Phi \neq \bar{\Phi}$ we set $X_{3}^{\prime}=\Phi-\rho\left(B C^{*}\right) X_{2} \rho\left(C B^{*}\right)=$ $(1-\Phi)\left(1-\bar{\Phi}(\Phi-\bar{\Phi}) E_{13}\right.$ and again obtain the full matrix algebra using $H E_{11}$ to obtain the needed elementary matrices. If $\Phi=\bar{\Phi}$, then, since $\Phi \neq 1$, we have $\Phi=-1$, hence $\alpha_{2}=\alpha_{3}=-1$ and so $\phi_{2} \neq \bar{\phi}^{2}$ since otherwise $\phi_{2}= \pm 1$ and $\alpha_{2}=\phi_{2}^{4}=1$. Thus we get $X_{3}^{\prime \prime}=\bar{\phi}_{2} X_{2}-\rho(A) X_{2} \rho\left(A^{*}\right)=4\left(\bar{\phi}_{2}-\phi_{2}\right) E_{13}$ with the factor being invertible. Again we are done.

Proposition 4.10. If $\Phi=1$ and not all $\alpha_{i}=\bar{\alpha}_{i}= \pm 1$, then the BH-algebra $\mathcal{B}_{\Theta}$ is equal to the full matrix algebra $\mathcal{B}_{\Theta}=M_{4}\left(\mathbb{\mathbb { T }}_{\Theta}^{3}\right)$.

Proof. As before this follows from an explicit representation of the generators $E_{i j}$ as expressions in $\mathcal{B}_{\Theta}$. Since not all $\alpha_{i}=1$, there must be some $\alpha_{i} \neq 1$. We will assume that $\alpha_{3} \neq 1$. The other calculations are symmetric. Again one has two cases. Either $\alpha_{2}=\bar{\alpha}_{2}$ or not. In the second case, we set $Y_{1}=H-\rho\left(B C^{*}\right) H \rho\left(C B^{*}\right)$ and $Y_{2}=\alpha_{2} Y_{1}=\rho\left(A C^{*}\right) Y_{1} \rho\left(A B^{*}\right)$. Put $Y_{3}=\alpha_{3} \bar{\phi}_{1} \bar{\phi}_{3}^{2} Y_{2}-\rho(C) H \rho\left(C^{*}\right)$ and $Y_{4}=$ $\bar{\phi}_{1} \phi_{2} Y_{3}-\rho(A) H \rho\left(A^{*}\right)=\left(1-\bar{\alpha}_{3}\right)\left(\alpha_{3}-\bar{\alpha}_{3}\right)\left(\alpha_{3} \bar{\phi}_{2} \bar{\phi}_{3}^{2}-\bar{\phi}_{2}^{2} \phi_{3}\right)\left(\bar{\phi}_{1} \phi_{2}-\alpha_{2}-\bar{\phi}_{2} \bar{\phi}_{2}^{2}\right) E_{43}$. Now as $\alpha_{3} \neq 1$ : $Y_{4} \neq 0$ if $\phi_{2} \neq 1$. If $\phi_{2}=1$, we must have $\phi_{1} \neq 1$ because otherwise $\phi_{3}$ would also be 1 . In this case, we proceed analogously to obtain $E_{24}$. In both cases we obtain the full matrix algebra following the strategy used in the previous proof.

Finally, we deal with the case that $\alpha_{2}=\bar{\alpha}_{2}$ : If $\alpha_{2}=1$ then $\alpha_{1}=\bar{\alpha}_{3}$. It follows that $\alpha_{1} \neq \bar{\alpha}_{1}$, and we are done by an analogous calculation. Indeed, if $\alpha_{1}=\bar{\alpha}_{1}$ then also $\alpha_{3}=\bar{\alpha}_{3}$ and all $\alpha_{i}$ are real, which we excluded.

If $\alpha_{2}=-1$, then $\alpha_{1}=-\bar{\alpha}_{3}$. It follows that $\alpha_{1} \neq \bar{\alpha}_{1}$, and we are done by an analogous calculation. If $\alpha_{1}=-\bar{\alpha}_{3}$, then $\alpha_{1}=\bar{\alpha}_{1}$ again means that all three $\alpha_{i}$ are real, which was excluded.

Proposition 4.11. If $\Phi=1$ and all of the $\alpha_{i}=1$, then the BH-algebra $\mathcal{B}_{\Theta}$ is $a$ proper subalgebra of the full matrix algebra $\mathcal{B}_{\Theta} \subsetneq M_{4}\left(\mathbb{T}^{3}\right)$.

Proof. We will show this using the character $\chi: \mathbb{T}^{3} \rightarrow \mathbb{C}$ defined by $\chi(A)=\chi(B)=$ $\chi(C)=1$. This character induces an algebra morphism $\bar{\chi}: M_{4}\left(\mathbb{T}^{3}\right) \rightarrow M_{4}(\mathbb{C})$, and we will show that $\bar{\chi}\left(\mathscr{B}_{\Theta}\right) \subsetneq M_{4}(\mathbb{C})$, which implies the result. We note that

$$
\bar{\chi}(H)=\left(\begin{array}{llll}
0 & 1 & 1 & 1 \\
1 & 0 & 1 & 1 \\
1 & 1 & 0 & 1 \\
1 & 1 & 1 & 0
\end{array}\right)=F-\text { Id } \quad \text { with } F=\left(\begin{array}{llll}
1 & 1 & 1 & 1 \\
1 & 1 & 1 & 1 \\
1 & 1 & 1 & 1 \\
1 & 1 & 1 & 1
\end{array}\right) .
$$


Since $\alpha_{i}=\phi_{i}^{4}$, all the $\phi_{i}$ must be fourth roots of unity and furthermore $\phi_{1} \phi_{2} \phi_{3}=1$. There are again three cases.

The first is that all $\phi_{i}=1$. Then $\bar{\chi}(\rho(A))=\bar{\chi}(\rho(B))=\bar{\chi}(\rho(C))=$ Id. Therefore $\bar{\chi}\left(\mathscr{B}_{\Theta}\right)=\langle\bar{\chi}(H)\rangle \subset M_{4}(\mathbb{C})$. Now $F^{n}=4^{n-1} F$ and so $\bar{\chi}(H)^{n}=$ id $+\left(\sum_{i=1}^{n}\left(\begin{array}{c}n \\ i\end{array}\right) 4^{i-1}\right) F$ so that $\bar{\chi}\left(\mathscr{B}_{\Theta}\right)$ is the two-dimensional subalgebra of $M_{4}(\mathbb{C})$ spanned by Id and $F$.

The second case is that all $\phi_{i}^{2}=1$ and only one $\phi_{i}=1$. We will assume that $\phi_{1}=1, \phi_{2}=-1, \phi_{3}=-1$. The rest of the cases are symmetric.

In this case the image of $\bar{\chi}$ is the 6-dimensional subalgebra of matrices of the form

$$
\left(\begin{array}{llll}
a & b & c & c \\
b & a & c & c \\
d & d & e & f \\
d & d & f & e
\end{array}\right) .
$$

Let $\mathscr{B}^{\prime}$ be the subalgebra above. It is an exercise to check that this is indeed a subalgebra. Then $\bar{\chi}(\rho(A))=\bar{\chi}(\rho(B))=\bar{\chi}(\rho(C))=\operatorname{diag}(1,1,-1,-1)$ so that $\bar{\chi}\left(\mathscr{B}_{\Theta}\right) \subset \mathscr{B}^{\prime}$. On the other hand, $P_{1}=E_{11}+E_{22}=\frac{1}{2}(\operatorname{Id}+\operatorname{diag}(1,1,-1,-1)) \epsilon$ $\bar{\chi}\left(\mathscr{B}_{\Theta}\right)$ and $P_{2}=E_{33}+E_{44}=\frac{1}{2}(\operatorname{Id}-\operatorname{diag}(1,1,-1,-1)) \in \bar{\chi}\left(\mathscr{B}_{\Theta}\right)$. And hence all the $P_{i} \bar{\chi}(H) P_{j}, i, j=1,2$, are in $\bar{\chi}\left(\mathscr{B}_{\Theta}\right)$. These are the $(2 \times 2)$ block matrices making up $\bar{\chi}(H)$. But the span of these matrices is precisely $\mathscr{B}^{\prime}$.

The final case is that exactly one $\phi_{i}^{2}=1$. We will treat the case $\phi_{1}=1, \phi_{2}=i$, $\phi_{3}=-i$. The rest is again symmetric. In this case $\bar{\chi}\left(\mathscr{B}_{\Theta}\right)$ is the 10 -dimensional matrix algebra of matrices of the form

$$
\left(\begin{array}{llll}
a & b & c & d \\
b & a & c & d \\
e & e & g & h \\
f & f & k & l
\end{array}\right)
$$

Let $\mathscr{B}^{\prime \prime}$ be the subalgebra above. Again it is an exercise to check that $\mathscr{B}^{\prime \prime}$ is indeed a subalgebra. In the current case we have $\bar{\chi}(\rho(A))=\bar{\chi}(\rho(B))=\bar{\chi}(\rho(C))=$ $\operatorname{diag}(1,1, i,-i)$, so it follows that $\bar{\chi}\left(\mathscr{B}_{\Theta}\right) \subset \mathscr{B}^{\prime \prime}$. Now $\bar{\chi}(\rho(A))^{2}=\operatorname{diag}(1,1,-1,-1)$ so that $P_{1}, P_{2}$ above are in $\bar{\chi}\left(\mathscr{B}_{\Theta}\right)$. Furthermore we have $\frac{-1}{2 i}\left(\bar{\chi}(\rho(A)) P_{2} \bar{\chi}(H) P_{2}-\right.$ $i P_{2} \bar{\chi}(H) P_{2}=E_{34}$ and $\frac{1}{2 i}\left(\bar{\chi}(\rho(A)) P_{2} \bar{\chi}(H) P_{2}=i P_{2} \bar{\chi}(H) P_{2}=E_{43}\right.$, which implies that $E_{33}, E_{34}, E_{43}, E_{44} \in \bar{\chi}\left(\mathscr{B}_{\Theta}\right)$. The other generators of $\mathscr{B}^{\prime}$ are now given by $P_{1}, P_{1} \bar{\chi}(H) P_{1}, P_{2} \bar{\chi}(H) P_{1}, E_{44} P_{2} \bar{\chi}(H) P_{1}, P_{1} \bar{\chi}(H) P_{2} E_{33}$ and $P_{1} \bar{\chi}(H) P_{2} E_{33}$, which are all in $\bar{\chi}\left(\mathscr{B}_{\Theta}\right)$.

The trickiest case is the case in which all the $\alpha_{i}$ are real and not all equal to 1.

Proposition 4.12. Let $\Phi=1$ and let all the $\alpha_{i}$ be real, but not all $\alpha_{i}=1$. If all the $\phi_{i}$ are different, we either have $\mathcal{B}_{\Theta}=M_{4}\left(\mathbb{T}_{\Theta}\right)$ or else $\mathcal{B}_{\Theta} \subsetneq M_{4}\left(\mathbb{T}_{\Theta}\right)$. 
Proof. Since not all $\alpha_{i}=1$, there must be exactly one $\alpha_{i}=1$ with the other two being equal to -1 . We will deal with the case $\alpha_{3}=1, \alpha_{2}=\alpha_{3}=-1$. The others are similar. Consider $X=H+\rho\left(B C^{*}\right) H \rho\left(C B^{*}\right)$. Then $X^{2}=12 E_{11}$ and $X_{2}=H E_{11}=E_{21}+E_{31}+E_{41}$. We know that $\phi_{1} \neq \phi_{2}, \phi_{1} \neq \phi_{3}$ and $X_{3}:=\phi_{1} X_{2}-\rho(A) X_{2} \rho\left(A^{*}\right)=\left(\phi_{1}-\phi_{2}\right) E_{31}+\left(\phi_{1}-\phi_{3}\right) E_{41}$. Here we get two cases.

If $\phi_{2} \neq \phi_{3}$, then we obtain $\phi_{2} X_{3}-\rho(A) X_{3} \rho\left(A^{*}\right)=\left(\phi_{2}-\phi_{3}\right)\left(\phi_{1}-\phi_{3}\right) E_{31}$, and we have successively $E_{31}, E_{41}, E_{21}$ and their transposes in $\mathcal{B}_{\Theta}$. But this is a set of generators.

In case $\phi_{2}=\phi_{3}$, we see that $P_{1}=\frac{1}{\phi_{1}-\phi_{2}} X_{2}=E_{31}+E_{41}$. Hence also $E_{12}, E_{21}, E_{22} \in \mathcal{B}_{\Theta}$. Furthermore, $P_{2}=P_{1} E_{12}=E_{32}+E_{42} \in \mathcal{B}_{\Theta}$ and $Q_{1}=$ $\mathrm{Id}-E_{11}-E_{22}=E_{33}+E_{44} \in \mathcal{B}_{\Theta}, Q_{2}=Q_{1} H Q_{1}$.

We shall use the morphism $\chi: \mathbb{T}_{\Theta}^{3} \rightarrow \mathrm{Cl}:=\operatorname{Cliff}\left(\left(\begin{array}{ll}1 & 0 \\ 0 & 1\end{array}\right)\right) \otimes \mathbb{C}$ given by $\chi(A)=$ $\chi(B)=e_{1}, \chi(C)=e_{2}$, where $e_{i}, i=1,2$, are the generators of the Clifford algebra which satisfy $e_{i}^{2}=1$ and $e_{1} e_{2}+e_{2} e_{1}=1$. The map $\chi$ induces an algebra morphism $\bar{\chi}: M_{4}\left(\mathbb{\mathbb { T }}_{\Theta}^{3}\right) \rightarrow \mathrm{Cl}$.

Claim: The image of $\bar{\chi}$ is given matrices of the form

$$
\left(\begin{array}{llll}
a & b & e & e \\
c & d & f & f \\
g & h & k & l \\
g & h & l & k
\end{array}\right)
$$

which is a free rank $10 \mathrm{Cl}$ proper submodule of $M_{4}(\mathrm{Cl})$ and in turn $\mathcal{B}_{\Theta}$ is a proper submodule of $M_{4}\left(\mathbb{\nabla}_{\Theta}^{3}\right)$.

To prove the claim, we again check both inclusions. Let $\mathscr{B}^{\prime}$ be the subalgebra above. Notice that $\mathrm{Id} \in \mathcal{B}^{\prime}$,

$$
\bar{\chi}(H)=\left(\begin{array}{cccc}
0 & 1 & 1 & 1 \\
1 & 0 & e_{1} & e_{1} \\
1 & e_{1} & 0 & e_{2} \\
1 & e_{1} & e_{2} & 0
\end{array}\right) \in \mathcal{B}^{\prime}
$$

$\bar{\chi} \rho(A)=\bar{\chi} \rho(B)=e_{1} \operatorname{diag}\left(1, \phi_{1}, \phi_{2}, \phi_{2}\right) \in \mathcal{B}^{\prime}, \bar{\chi} \rho(C)=e_{2} \operatorname{diag}\left(1, \phi_{1}, \phi_{2}, \phi_{2}\right) \in$ $\mathscr{B}^{\prime}$ so that $\bar{\chi}\left(\mathscr{B}_{\Theta}\right) \subset \mathscr{B}^{\prime}$. On the other hand the algebra $\mathscr{B}^{\prime}$ is generated by $E_{11}, E_{12}$, $E_{21}, E_{22}, P_{1}, P_{1}^{T}, P_{2}, P_{2}^{T}, Q_{1}, e_{2} \bar{\chi}\left(Q_{2}\right)=E_{34}+E_{43}$, which are all in $\bar{\chi}\left(\mathcal{B}_{\Theta}\right)$ so that $\mathcal{B}_{\Theta} \subset \bar{\chi}\left(\mathscr{B}_{\Theta}\right)$.

Theorem 4.13. If $\Phi \neq 1$ or $\Phi=1$ and at least one $\alpha_{i} \neq 1$ and all $\phi_{i}$ are different, then $\mathscr{B}_{\Theta}=M_{4}\left(\mathbb{\mathbb { T }}_{\Theta}^{3}\right)$ and $K\left(\mathscr{B}_{\Theta}\right)=\mathbb{Z}^{4}$.

If $\phi_{i}=1$ for all $i$, then $K\left(\mathcal{B}_{\Theta}\right)=K(X)$, where $X$ is the cover of the 3-torus given by Theorem 3.21 .

Proof. The only thing that remains to be proved is that $H$ is generic. Indeed this can be done by direct computation. We will not give the eigenvalues here as they are 
quite long expressions. But notice that for $\chi(A)=-1, \chi(B)=1, \chi(C)=-1$ the matrix $\bar{\chi}(H)$ has the four distinct eigenvalues $\pm \sqrt{5}, \pm 1$.

Acknowledgments. We would like to thank J. Bellissard, M. Dadarlat, M. Kontsevich, M. Marcolli and A. Schwarz for enlightening discussions. Foremost, we wish to thank H. W. Hillhouse for sparking the interest in this project and many discussions.

RK thankfully acknowledges support from NSF DMS-0805881 and the Humboldt Foundation. He also thanks the Institut des Hautes Etudes Scientifiques, the MaxPlanck-Institute for Mathematics in Bonn and the Institute for Advanced Study for their support as well as the University of Hamburg for its hospitality. While at the IAS, RK's work was supported by the NSF under agreement DMS-0635607.

BK thanks the Department of Physics at Princeton University for its hospitality and thankfully acknowledges support from the NSF under the grant PHY-0969689.

Any opinions, findings and conclusions or recommendations expressed in this material are those of the authors and do not necessarily reflect the views of the National Science Foundation.

\section{References}

[1] N. W. Ashcroft and N. D. Mermin, Solid state physics. Holt, Rinehart and Winston, New York 1976.

[2] J. Bellissard, Gap labelling theorems for Schrödinger operators. In From number theory to physics (Les Houches, 1989), Springer-Verlag, Berlin 1992, 538-630. Zbl 0833.47056 MR 1221111

[3] J. Bellissard, A. van Elst, and H. Schulz-Baldes, The noncommutative geometry of the quantum Hall effect. J. Math. Phys. 35 (1994), 5373-5451. Topology and physics. Zbl 0824.46086 MR 1295473

[4] A. L. Carey, K. C. Hannabuss, V. Mathai, and P. McCann, Quantum Hall effect on the hyperbolic plane. Comm. Math. Phys. 190 (1998), 629-673. Zbl 0916.46057 MR 1600480

[5] A. Connes, Noncommutative geometry. Academic Press, San Diego, CA, 1994. Zbl 0818.46076 MR 1303779

[6] G. A. Elliott, On the $K$-theory of the $C^{*}$-algebra generated by a projective representation of a torsion-free discrete abelian group. In Operator algebras and group representations, Vol. I, Monogr. Stud. Math. 17, Pitman, Boston 1984, 157-184. Zbl 0542.46030 MR 731772

[7] G. A. Elliott and H. Li, Strong Morita equivalence of higher-dimensional noncommutative tori. II. Math. Ann. 341 (2008), 825-844. Zbl 1153.46045 MR 2407328

[8] K. Grosse-Brauckmann, On gyroid interfaces. J. Colloid Interface Sci. 187 (1997), 418-428.

[9] K. Große-Brauckmann and M. Wohlgemuth, The gyroid is embedded and has constant mean curvature companions. Calc. Var. Partial Differential Equations 4 (1996), 499-523. Zbl 0930.53009 MR 1415998 
[10] D. A. Hajduk, P. E. Harper, S. M. Gruner, C. C. Honeker, G. Kim, E. L. Thomas, and L. J. Fetters, The gyroid: a new equilibrium morphology in weakly segregated diblock copolymers. Macromolecules 27 (1994), 4063-4075.

[11] T. Hahn (ed.), International tables for crystallography. Vol. A, 5. ed., reprinted with corrections, Springer-Verlag, Dordrecht 2005.

[12] P. G. Harper, Single band motions of conduction electrons in a uniform magnetic field. Proc. Phys. Soc. A 68 (1955), 874-878.

[13] S. Khlebnikov and H. W. Hillhouse, Electronic structure of double-gyroid nanostructured semiconductors: Perspectives for carrier multiplication solar cells. Phys. Rev. B 80 (2009), 115316.

[14] C. A. Lambert, L. H. Radzilowski, and E. L. Thomas, Triply periodic level surfaces as models for cubic tricontinous block copolymer morphologies. Phil. Trans. R. Soc. Lond. A 354 (1996), 2009-2023.

[15] M. Marcolli and V. Mathai, Towards the fractional quantum Hall effect: a noncommutative geometry perspective. In Noncommutative geometry and number theory, Aspects Math. E37, Vieweg, Wiesbaden 2006, 235-261. Zbl 1105.81068 MR 2327308

[16] G. Panati, H. Spohn, and S. Teufel, Effective dynamics for Bloch electrons: Peierls substitution and beyond. Comm. Math. Phys. 242 (2003), 547-578. Zbl 1058.81020 MR 2020280

[17] M. Pimsner and D. Voiculescu, Exact sequences for $K$-groups and Ext-groups of certain cross-product $C^{*}$-algebras. J. Operator Theory 4 (1980), 93-118. Zbl 0474.46059 MR 587369

[18] M. A. Rieffel, $C^{*}$-algebras associated with irrational rotations. Pacific J. Math. 93 (1981), 415-429. Zbl 0499.46039 MR 623572

[19] M. A. Rieffel and A. Schwarz, Morita equivalence of multidimensional noncommutative tori. Internat. J. Math. 10 (1999), 289-299. Zbl 0968.46060 MR 1687145

[20] T. Sunada, A discrete analogue of periodic magnetic Schrödinger operators. In Geometry of the spectrum (Seattle, WA, 1993), Contemp. Math. 173, Amer. Math. Soc., Providence, RI, 1994, 283-299. Zbl 0805.47028 MR 1298211 (95i:58185)

[21] A. H. Schoen, Infinite periodic minimal surfaces without self-intersections. NASA Technical Note D-5541. Washington, DC, 1970. Zbl 1071.53507

[22] M. F. Schulz, F. S. Bates, K. Almdal, and K. Mortensen, Epitaxial relationship for hexagonal-to-cubic phase transition in a book copolymer mixture. Phys. Rev. Lett. 73 (1994), 86-89.

[23] V. N. Urade, T.-C. Wei, M. P. Tate, J. D. Kowalski, and H. W. Hillhouse, Nanofabrication of double-gyroid thin films. Chem. Mater. 19 (2007), 768-777.

Received October 5, 2010

R. M. Kaufmann, Department of Mathematics, Purdue University, 150 N. University Street, West Lafayette, IN 47907-2067, U.S.A.

E-mail: rkaufman@math.purdue.edu 
S. Khlebnikov, Department of Physics, Purdue University, 525 Northwestern Avenue, West Lafayette, IN 47907-2036, U.S.A.

E-mail: skhleb@physics.purdue.edu

B. Wehefritz-Kaufmann, Department of Mathematics and Department of Physics, $150 \mathrm{~N}$. University Street, Purdue University, West Lafayette, IN 47907-2067, U.S.A.

E-mail: ebkaufma@math.purdue.edu 